\title{
Algorithms to Detect and Rectify Multiplicative and Ordinal Inconsistencies of Fuzzy Preference Relations
}

\author{
Yejun Xu , Mengqi Li, Francisco Javier Cabrerizo, Francisco Chiclana, Enrique Herrera-Viedma
}

\begin{abstract}
Consistency, multiplicative and ordinal, of fuzzy preference relations (FPRs) is investigated. The geometric consistency index (GCI) approximated thresholds are extended to measure the degree of consistency for an FPR. For inconsistent FPRs, two algorithms are devised (1) to find the multiplicative inconsistent elements, and (2) to detect the ordinal inconsistent elements. An integrated algorithm is proposed to improve simultaneously the ordinal and multiplicative consistencies. Some examples, comparative analysis, and simulation experiments are provided to demonstrate the effectiveness of the proposed methods.
\end{abstract}

Index Terms-FPRs; Multiplicative consistency (MC); Ordinal consistency (OC); Inconsistent elements.

\section{INTRODUCTION}

In multiple criteria decision-making problems, pairwise comparisons on alternatives are usually used by decision makers (DMs) to express their preference information [1-3], which can be modelled mathematically with a preference relation (PR). Multiplicative PRs (MPRs) [4-9] and fuzzy PRs (FPRs) [10-13] are widely used for numerical PRs. A key drawback of pairwise comparison is that DMs fail to consider the global relationship of alternatives because they focus on two alternatives at a time. This may end in the provision of inconsistent preferences[14], especially in the presence of a large number of alternatives. Thus, in decision making scenarios with PRs, it is sensible to ensure that preferences are consistent before making the final decision.

Consistency of preferences generally has two types: ordinal consistency (OC) [15-19] and cardinal consistency (CC) [18,

This work was partly supported by the National Natural Science Foundation of China (NSFC) under Grants (No. 71871085, 71471056, 71433003), the financial support of the grant TIN2016- 75850-R from the Spanish Ministry of Innovation and Universities.

Yejun $\mathrm{Xu}$ is with the Business School, Hohai University, Nanjing, 211100, PR China (Corresponding author, E-mail: xuyejohn@163.com).

Mengqi Li is with the Business School, Hohai University, Nanjing, 211100, PR China (E-mail: sdbzlm7@163.com).

Francisco Javier Cabrerizo is with the Andalusian Research Institute in Data Science and Computational Intelligence, University of Granada, Granada 18071, Spain (E-mail: cabrerizo@decsai.ugr.es)

Francisco Chiclana is with the Andalusian Research Institute in Data Science and Computational Intelligence, University of Granada, Granada 18071, Spain, and also with Institute of Artificial Intelligence, School of Computer Science and Informatics, De Montfort University, Leicester, LE1 9BH, UK (E-mail: chiclana@dmu.ac.uk)

Enrique Herrera-Viedma is with the Andalusian Research Institute in Data Science and Computational Intelligence, University of Granada, Granada 18071, and also with Department of Electrical and Computer Engineering, Faculty of Engineering, King Abdulaziz University, Jeddah 21589, Saudi Arabia (Corresponding author, e-mail: viedma@decsai.ugr.es). 
20-22]. The OC, which is based on the concept of the weak transitivity [23], is defined as follows: "if alternative $x_{i}$ is preferred to alternative $x_{j}$ and alternative $x_{j}$ is preferred to alternative $x_{k}$, then alternative $x_{i}$ should be preferred to alternative $x_{k}$ ". This type of consistency is considered to be the minimum requirement to be fulfilled by a 'consistent' PR [4, $21,24]$. A stronger concept than $\mathrm{OC}$ is $\mathrm{CC}$ because it requires in addition that the "intensity with which the preference is expressed transits through the sequence of objects in comparison" [4].

In the Analytic Hierarchy Process (AHP), Saaty [4] introduced the notions of perfect consistency and acceptable consistency. This was based on the consistency ratio (CR) between the consistency index (CI) of an MPR and the average CIs of a large number of random generated MPRs of the same order. An MPR with $C R<0.1$ is considered of acceptable consistency. However, acceptable consistency does not imply OC as illustrated by Kwiesielewicz and van Uden [17] where an MPR with CR smaller than 0.1 was shown to be ordinally inconsistent, i.e., it contained inconsistent elements.

There are also studies on the consistency of FPRs that deal with different types of inconsistencies: OC [16, 25], additive consistency (AC) [16, 26-28] and multiplicative consistency (MC) [13, 29]. Ma, et al. [30] introduced a method to detect and repair the inconsistency of strict FPRs. Li, et al. [22] reviewed the AC of FPRs. Xu, et al. [15] developed an OC index (OCI) to measure the level of OC of FPRs and an algorithm to eliminate unreasonable 3-cycles in the relation digraph. Xia, et al. [13] adapted the geometric consistency index (GCI) for MPRs proposed by Aguarón and Moreno-Jiménez [31] to the case of FPRs, which was the base of a method to improve their MC. However, $\mathrm{Xu}$, et al. [29] provided an example where contradictory preference values may still be present in an FPR that fulfils the MC test, which suggests that MC on its own is not sufficient to assure the consistency of FPRs. Thus, it is clear that there is a need for a study that focus on both the OC and $\mathrm{CC}$ at the same time.

There conflict between AC and the scale for measuring FPR values $([0,1])$ led Chiclana, et al. [20] to propose a functional modelling of consistency of FPRs, that under the conditions of monotonicity and continuity was proved to be verified by a representable uninorm with strict negator operator $N(x)=1-x$. Because the MC is an AND-like representable uninorm with the above strict negator operator, Chiclana, et al. [20] concluded that MC is the most appropriate property for modelling CC of FPRs. Meanwhile, few researches have investigated both the $\mathrm{OC}$ and the MC at the same time. Because the OC can ensure the rationality and the MC can measure the level of consistency and lead to more consistent FPRs. Xu, et al. [29] developed some methods to modify the ordinal and multiplicative inconsistencies for FPRs. However, their improvement methods have two steps: ordinal inconsistency modification step, and multiplicative inconsistency modification step. The latter step aims at improving multiplicative consistency by adjusting the elements that contributes most to the multiplicative inconsistency, which it is followed by a checking of whether the adjustment destroys ordinal consistency. If the modified FPR does not have ordinal consistency, the method requires a further check and adjustment of the element second most contributor to multiplicative inconsistency. These are obviously complex and time-consuming methods. Although the OC 
and MC of FPRs have been deeply investigated, there are still some open questions to be answered:

(1) In the existing work (for example, Refs. [13, 29]), the GCI threshold is usually set artificially and may not be reasonable. Thus, the following question needs to be addressed: is there a reasonable threshold for GCI to measure the level of MC?

(2) One of the two types of consistency discussed is not enough to ensure the rationality of the FPRs, while improving the two types of consistency separately will make the calculation process time-consuming and will also distort the information greatly. Thus, the following question requires also consideration: Can the OC and MC be improved simultaneously with a unique single method?

This paper aims at answering the above two research questions and fill a gap in the current research knowledge. This paper extends GCI to measure the MC of an FPR. A new method to detect and measure the OC is also devised. Finally, an integrated algorithm to find the inconsistent elements to improve the $\mathrm{OC}$ and $\mathrm{MC}$ simultaneously is proposed. Monte Carlo simulation are also provided to show the effectiveness and advantages of the proposed methods.

The remainder of the paper is organized as follows. Section II contains basic concepts about consistency of FPRs that will be used in subsequent sections. Section III proposes an MC-based algorithm to identify FPRs multiplicative inconsistent elements. Section IV presents a new index to measure the OC level and an algorithm to search the ordinal inconsistent elements of FPRs. Section V presents an algorithm to adjust the FPR inconsistent elements, while examples of its application and a comparison with existing methods in the literature to demonstrate the effectiveness of the proposed method are provided in Section VI. Finally, in Section VII conclusions are given.

\section{Preliminaries}

This section provides the basic definition related to FPRs needed in the following sections.

For simplicity, let $N=\{1,2, \ldots, n, n>2\}$ and $X=\left\{x_{i}, i \in N\right\}$ be a finite set of alternatives. In multi-attribute decision making problems, DMs aim to get ranking of alternatives based on the provided information which is assumed to be in the form of FPRs.

Definition 1 [11]: "An FPR $R$ on a set of alternatives $X$ is a fuzzy set on the cartesian product set $X \times X$, which is characterized by a membership function $\mu_{R}: X \times X \rightarrow[0,1]$, which is represented by a $n \times n$ matrix $R=\left(r_{i j}\right)_{n \times n}$, with element $r_{i j}$ interpreted as the preference degree of the alternative $x_{i}$ over the alternative $x_{j}$. A value of $r_{i j}=0.5$ indicates indifference between $x_{i}$ and $x_{j}\left(x_{i} \sim x_{j}\right)$; When $0.5<r_{i j} \leq 1$, then $x_{i}$ is strictly preferred to $x_{j} \quad\left(x_{i} \succ x_{j}\right)$ with $r_{i j}=1$ indicating absolute preference of $x_{i}$ over $x_{j}$; while $0 \leq r_{i j}<0.5$ indicates that $x_{j}$ is strictly preferred to $x_{i}\left(x_{i} \prec x_{j}\right)$ with $r_{i j}=0$, indicating absolute preference of $x_{j}$ over $x_{i}$ ”.

This interpretation underlines a reciprocity property of preference often assumed to be verified, as it is the case in the 
present paper:

$$
r_{i j}+r_{j i}=1, \quad r_{i i}=0.5, \quad r_{i j} \in[0,1], \forall i, j \in N
$$

\section{A. Ordinal Consistency of FPRS}

The OC of FPRs is described as follows:

Definition 2 [15]: "An FPR $R=\left(r_{i j}\right)_{n \times n}$ is OC when for all $i, j, k \in N, i \neq j \neq k$, the following properties are verified:

(1) if $\left[r_{i k}>0.5, r_{k j} \geq 0.5\right]$, or $\left[r_{i k} \geq 0.5, r_{k j}>0.5\right]$ then $r_{i j}>0.5$;

(2) if $\left[r_{i k}=0.5\right.$ and $\left.r_{k j}=0.5\right]$ then $r_{i j}=0.5 \%$.

$\mathrm{Xu}$, et al. [15] applied graph theory to investigate the OC of FPRs.

Definition 3 [15]: "The adjacency matrix of an FPR $R=\left(r_{i j}\right)_{n \times n}$ is:

$$
B=\left(b_{i j}\right)_{n \times n} ; \quad b_{i j}= \begin{cases}1, & r_{i j} \geq 0.5, i \neq j \\ 0, & \text { otherwise }\end{cases}
$$

The digraph of the FPR $R$ is denoted by $G=(X, A)$, where the set of alternatives $X$ is the node set and $r_{i j}>0.5$ $A=\left\{\left(x_{i}, x_{j}\right) \mid i \neq j \wedge r_{i j} \geq 0.5\right\}$ is the directed arc set. When $r_{i j}=0.5, i \neq j$, it is also $r_{j i}=0.5$ and therefore there exist two directed arcs between $x_{i}$ and $x_{j}$ (one from $x_{i}$ to $x_{j}$, and another from $x_{j}$ to $x_{i}$ )."

Remark 1: It is easy to prove that if an FPR $R$ violates OC, then there must exist at least one directed 3-cycle in the digraph $G$ of $R$, i.e. $x_{i} \rightarrow x_{j} \rightarrow x_{k} \rightarrow x_{i}$. Therefore, the OC of FPRs requires directed 3-cycles to be identified and eliminated.

\section{B. Multiplicative consistency of FPRs}

MC refers to the multiplicative transitivity property of preferences:

Definition 4 [11]: “An FPR $R=\left(r_{i j}\right)_{n \times n}$ is MC if it satisfies:

$$
r_{i j} r_{j k} r_{k i}=r_{j i} r_{k j} r_{i k}, \forall i, j, k \in N
$$

Which is referred to as the multiplicative transitivity property".

Remark 2: Eq.(3) requires that $r_{i j}>0, \forall i, j$, and therefore it can be rewritten as:

$$
\frac{r_{i j}}{r_{j i}}=\frac{r_{i k}}{r_{k i}} \cdot \frac{r_{k j}}{r_{j k}}, \forall i, j, k \in N
$$

The value $r_{i j} / r_{j i}$ represents the ratio of the preference power for $x_{i}$ to that of $x_{j}$, and it is interpreted as follows: $x_{i}$ is $r_{i j} / r_{j i}$ times preferred to $x_{j}$. This value will be referred to as the direct judgement of alternative $x_{i}$ over alternative $x_{j}$, while $\frac{r_{i k}}{r_{k i}} \cdot \frac{r_{k j}}{r_{j k}}$ will be referred to as the indirect judgement of alternative $x_{i}$ over alternative $x_{j}$ via (though) alternative 
$x_{k}$.

In order to measure the level of consistency, Aguarón and Moreno-Jiménez [31] proposed the geometric consistency index $(G C I)$ for reciprocal MPRs $A=\left(a_{i j}\right)_{n \times n}\left(a_{i j} \cdot a_{j i}=1\right)$ :

$$
G C I(A)=\frac{2}{(n-1)(n-2)} \sum_{i<j}\left(\log a_{i j}-\log v_{i}+\log v_{j}\right)^{2}
$$

where $v=\left(v_{1}, v_{2}, \cdots, v_{n}\right)^{T}$ is the following priority vector derived from $A$ :

$$
v_{i}=\left(\prod_{j=1}^{n} a_{i j}\right)^{1 / n} / \sum_{j=1}^{n}\left(\prod_{j=1}^{n} a_{i j}\right)^{1 / n} ; \sum_{i=1}^{n} v_{i}=1 \text { and } v_{i}>0, i \in N
$$

According to the theoretical relation between CR and the GCI, Aguarón and Moreno-Jiménez [31] provided the below approximated threshold values for GCI (Table I).

TABLE I. THE APPROXIMATED THRESHOLDS FOR GCI

\begin{tabular}{ccccc}
\hline$C R$ & 0.01 & 0.05 & 0.1 & 0.15 \\
\hline$\overline{G C I}(n=3)$ & 0.0314 & 0.1573 & 0.3147 & 0.4720 \\
$\overline{G C I}(n=4)$ & 0.0352 & 0.1763 & 0.3526 & 0.5289 \\
$\overline{G C I}(n>4)$ & $\sim 0.037$ & $\sim 0.185$ & $\sim 0.370$ & $\sim 0.555$ \\
\hline
\end{tabular}

The following transformation provides a route to derive an MPR $A=\left(a_{i j}\right)_{n \times n}$ from an FPR $R=\left(r_{i j}\right)_{n \times n}[32]$ :

$$
a_{i j}=\frac{r_{i j}}{r_{j i}}
$$

Motivated by Aguarón and Moreno-Jiménez [31], Xia, et al. [13] adopted the GCI to the case of FPRs.

Definition 5 [13]: "Let $R=\left(r_{i j}\right)_{n \times n}$ be an FPR, and $w=\left(w_{1}, w_{2}, \cdots, w_{n}\right)^{T}$ be the priority vector derived from $R$ satisfying $\sum_{i=1}^{n} w_{i}=1, w_{i}<1$ and $w_{i}>0, i \in N$, then the $G C I$ of $R$ is given by

$$
G C I(R)=\frac{2}{(n-1)(n-2)} \sum_{i<j}\left(\ln r_{i j}-\ln r_{j i}-\ln w_{i}+\ln w_{j}\right)^{2}
$$

When $G C I(R)$ is zero, the FPR $R$ is of perfect MC. The smaller the value of $G C I(R)$, the better the consistency of $R$ ".

In practical situations, the DMs' FPRs are not of perfect MC. In these cases, a threshold value $\overline{G C I}$ is set when $G C I(R) \leq \overline{G C I}$ the FPR $R$ is considered of acceptable MC. Xia, et al. [13] did not provide $\overline{G C I}$ threshold values, although it is obvious from Eq.(5) and Eq.(6) that the threshold values of Table I for MPRs can be used to check acceptable MC for FPRs.

Given an FPR, checking its acceptable MC requires the computation of its priority vector in the first place. Wang and Fan [33] proposed the following logarithmic least squares model to obtain the priority vector from an FPR:

$$
\text { (M-1) } \min J=\sum_{i=1}^{n} \sum_{j=1}^{n}\left(\ln r_{i j}-\ln r_{j i}-\ln w_{i}+\ln w_{j}\right)^{2}
$$




$$
\text { s.t. } \sum_{i=1}^{n} w_{i}=1,0<w_{i}<1, i \in N
$$

The solution to (M-1) was also given by Wang and Fan [33]:

$$
w_{i}=\frac{\left(\prod_{j=1}^{n} \frac{r_{i j}}{r_{j i}}\right)^{1 / n}}{\sum_{i=1}^{n}\left(\prod_{j=1}^{n} \frac{r_{i j}}{r_{j i}}\right)^{1 / n}}, i \in N
$$

It coincides, as expected, with the one given by Aguarón and Moreno-Jiménez [31] for MPRs using Eq.(6).

$\mathrm{Xu}$, et al. [29] illustrated with some examples that FPR may still contain contradictory judgments in terms of OC but be regarded as MC. Thus, to study consistency of preferences properly MC is not sufficient on its own and OC should also be considered. This is the focus of Section IV where an effective method to measure OC of FPRs is put forward. Before this can be done, in next section the problem of detecting multiplicative inconsistency is first tackled.

\section{Multiplicative InCONSISTENCY Detection FOR FPRS}

First, measuring the 'MC degree' of FPRs can help devising effective methods to detect its most inconsistent elements. Subsequently, we can improve its consistency.

\section{A. The Multiplicative Inconsistency of FPRs}

The equality $r_{i j} / r_{j i}=\left(r_{i k} / r_{k i}\right) \cdot\left(r_{k j} / r_{j k}\right)$ ( for all $\left.i, j, k \in N\right)$ holds for an FPR $R=\left(r_{i j}\right)_{n \times n}$ of perfect MC. This property can be explained intuitively in Fig. 1, which combines direct and indirect judgments on a judgment scale.

If $R$ is of perfect MC, all indirect judgments $\left(r_{i k} / r_{k i}\right) \cdot\left(r_{k j} / r_{j k}\right)$ would be located in the blue dot of Fig. 1. Otherwise, there would be indirect judgments located on the right of the blue dot (as the two provided in Fig.1), which indicate preference ratios higher than the direct judgment, and/or on the left of the blue dot (as the one indirect in Fig. 1), which indicates preference ratios lower than the direct judgment.

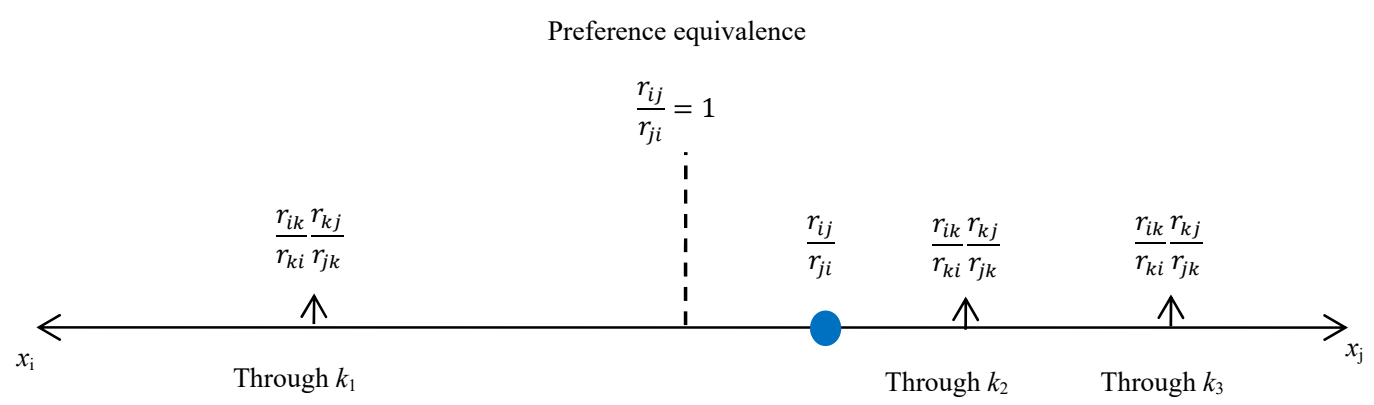

Fig. 1. The direct and indirect judgments on a judgment scale

From Fig. 1, if $R$ is not MC, then the direct value $r_{i j} / r_{j i}$ and the indirect preference value $\left(r_{i k} / r_{k i}\right) \cdot\left(r_{k j} / r_{j k}\right)$ may be diversely scattered and their deviation is measured as follows: 


$$
\delta\left(\frac{r_{i j}}{r_{j i}}, \frac{r_{i k}}{r_{k i}} \cdot \frac{r_{k j}}{r_{j k}}\right)=\left|\log \left(\frac{r_{i j}}{r_{j i}}\right)-\log \left(\frac{r_{i k}}{r_{k i}} \cdot \frac{r_{k j}}{r_{j k}}\right)\right|
$$

or

$$
\delta\left(\frac{r_{i j}}{r_{j i}}, \frac{r_{i k}}{r_{k i}} \cdot \frac{r_{k j}}{r_{j k}}\right)=\left(\log \left(\frac{r_{i j}}{r_{j i}}\right)-\log \left(\frac{r_{i k}}{r_{k i}} \cdot \frac{r_{k j}}{r_{j k}}\right)\right)^{2}
$$

Remark 3: Clearly an FPR is MC if and only if the deviation values from Eq.(9) to Eq.(10) are 0 for all $i, j, k \in N$. Meanwhile, if a particular deviation value associated with $r_{i j} / r_{j i}$ is not 0 , then we can say that $r_{i j}$ is an inconsistent element of the FPR.

In order to measure the inconsistency degree of an FPR and detect the multiplicative inconsistent elements, we first aggregate the above deviations values:

$$
\theta_{i j}=\frac{1}{n-2} \sum_{k=1, k \neq i, k \neq j}^{n} \delta\left(\frac{r_{i j}}{r_{j i}}, \frac{r_{i k}}{r_{k i}} \cdot \frac{r_{k j}}{r_{j k}}\right), \forall i, j, k \in N
$$

Alternative approaches to define $\theta_{i j}$ are possible, such as the worst deviation approach:

$$
\theta_{i j}=\max _{k \neq i, k \neq j}\left(\delta\left(\frac{r_{i j}}{r_{j i}}, \frac{r_{i k}}{r_{k i}} \cdot \frac{r_{k j}}{r_{j k}}\right)\right), \forall i, j, k \in N
$$

or the geometric mean approach

$$
\theta_{i j}=\left(\prod_{k=1, k \neq i, k \neq j}^{n} \delta\left(\frac{r_{i j}}{r_{j i}}, \frac{r_{i k}}{r_{k i}} \cdot \frac{r_{k j}}{r_{j k}}\right)\right)^{\frac{1}{n-2}}, \forall i, j, k \in N
$$

Different definitions of $\theta_{i j}$ and $\delta$ would result in different values of $\theta_{i j}$, which may lead to different results and different formulas in practical problems. In any case, in the symmetrical matrix $\Theta=\left(\theta_{i j}\right)_{n \times n}\left(\theta_{i j}=\theta_{j i}\right)$, the larger the value $\theta_{i j}$, the more inconsistent the element $r_{i j}$ is. Thus, the most inconsistent element of an FPR will correspond to the largest value in the matrix $\Theta=\left(\theta_{i j}\right)_{n \times n}$. Thus, the following algorithm (Algorithm 1) is proposed.

Algorithm 1. Let $R=\left(r_{i j}\right)_{n \times n}$ be an FPR, and $\overline{G C I(R)}$ a consistency threshold value from Table I.

Step 1. Compute $G C I(R)$ using Eq.(7). If $G C I(R) \leq \overline{G C I(R)}$, then $R$ has acceptable MC and go to Step 5. Otherwise, continue with the next step.

Step 2. Compute the deviation $\delta$ using Eq.(9) or Eq.(10).

Step 3. Compute $\theta_{i j}$ and establish the matrix $\Theta=\left(\theta_{i j}\right)_{n \times n}$ according to one of Eq.(11) to Eq.(13).

Step 4. Find the value $\theta_{i_{m} j_{m}}=\max _{i, j}\left\{\theta_{i j}\right\}$ in matrix $\Theta=\left(\theta_{i j}\right)_{n \times n}$, then the corresponding $r_{i_{m} j_{m}}$ is the most multiplicative 
inconsistent element in the FPR $R$.

Step 5. End.

\section{B. The Accumulation of Multiplicative Inconsistent Elements}

Given an FPR, the accumulation of the inconsistency values associated with its respective elements can be considered as its multiplicative inconsistency degree:

$$
\theta=\frac{1}{n(n-1)} \sum_{i=1}^{n} \sum_{j=1}^{n} \theta_{i j}
$$

Alternative expression for $\theta$, such as the worst deviation is stated below

$$
\theta=\max _{i, j}\left(\theta_{i j}\right)
$$

Depending on the different mathematical formulations used for $\delta$ and $\theta_{i j}, \theta$ may result in different values. In any case, when $\theta=0, R$ will be of perfect MC; while the smaller the value of $\theta$, the more consistent it will be.

As we know, $G C I$ is equivalent to $C I$ in that it is used to measure the degree of inconsistency of $R$, but it cannot identify inconsistent elements conveniently and intuitively. Therefore, after measuring the degree of inconsistency by GCI, if $G C I(R)>\overline{G C I(R)}, \Theta=\left(\theta_{i j}\right)_{n \times n}$ can be used to further locate the FPR multiplicative inconsistent elements. The following example illustrates this process.

Example 1: Assume a DM gives the following FPR (adapted from [15, 34]):

$$
R_{1}=\left[\begin{array}{llll}
0.5 & 0.7 & 0.9 & 0.5 \\
0.3 & 0.5 & 0.6 & 0.7 \\
0.1 & 0.4 & 0.5 & 0.8 \\
0.5 & 0.3 & 0.2 & 0.5
\end{array}\right]
$$

Algorithm 1 is run to compute the multiplicative inconsistent degree of $R_{1}$ and to identify its most MC element. Eq.(8) results in $w=(0.4697,0.2428,0.1619,0.1255)^{T}$. From Eq.(7), it is noticed that $G C I\left(R_{1}\right)=1.4581>0.3526$, and consequently $R_{1}$ is not of acceptable MC. Using Eq.(9) to Eq.(13), the corresponding matrix $\Theta=\left(\theta_{i j}\right)_{n \times n}$ are obtained

\begin{tabular}{|c|c|c|c|c|c|c|c|c|}
\hline & \multicolumn{4}{|c|}{$\delta$ (By Eq.(9)) } & \multicolumn{4}{|c|}{$\delta$ (By Eq.(10)) } \\
\hline $\begin{array}{c}\Theta \\
\text { (By Eq.(11)) }\end{array}$ & {$\left[\begin{array}{c}0 \\
1.3195 \\
2.2640 \\
2.6391\end{array}\right.$} & $\begin{array}{c}1.3195 \\
0 \\
0.9445 \\
1.3195\end{array}$ & $\begin{array}{c}2.2640 \\
0.9445 \\
0 \\
2.2640\end{array}$ & $\left.\begin{array}{c}2.6391 \\
1.3195 \\
2.2640 \\
0\end{array}\right]$ & $\begin{array}{c}0 \\
1.8818 \\
6.8668 \\
7.8566\end{array}$ & $\begin{array}{c}1.8818 \\
0 \\
0.8920 \\
1.8818\end{array}$ & $\begin{array}{c}6.8668 \\
0.8920 \\
0 \\
6.8668\end{array}$ & $\left.\begin{array}{c}7.8566 \\
1.8818 \\
6.8668 \\
0\end{array}\right]$ \\
\hline $\begin{array}{c}\Theta \\
\text { (By Eq.(12)) }\end{array}$ & {$\left[\begin{array}{c}0 \\
1.6946 \\
3.5835 \\
3.5835\end{array}\right.$} & $\begin{array}{c}1.6946 \\
0 \\
0.9445 \\
1.6946\end{array}$ & $\begin{array}{c}3.5835 \\
0.9445 \\
0 \\
3.5835\end{array}$ & $\left.\begin{array}{c}3.5835 \\
1.6946 \\
3.5835 \\
0\end{array}\right]$ & {$\left[\begin{array}{c}0 \\
2.8717 \\
12.8416 \\
12.8416\end{array}\right.$} & $\begin{array}{c}2.8717 \\
0 \\
0.8920 \\
2.8717\end{array}$ & $\begin{array}{c}12.8416 \\
0.8920 \\
0 \\
12.8416\end{array}$ & $\left.\begin{array}{c}12.8416 \\
2.8717 \\
12.8416 \\
0\end{array}\right]$ \\
\hline
\end{tabular}
and shown in Table II:

TABLE II. THE MATRIX $\Theta$ OBTAINED By DIFFERENT DEFINITIONS OF $\theta_{i j}$ AND $\delta$. 


\section{ORDINAL INCONSISTENCY DETECTION FOR FPRS}

This section introduces first some basic concepts of OC of FPRs. Then, an algorithm is provided to construct a matrix to locate ordinally inconsistent elements and the ordinal inconsistent matrix of an FPR, respectively. Some properties of the ordinally inconsistent matrix are also investigated.

\section{A. The Ordinal Inconsistency of FPRs}

The OC of FPRs directly affects the alternatives ranking. As discussed in Section 2, an FPR that passes the test of consistency $(G C I)$ does not guarantee its OC. Thus, an effective method to determine the level of OC is required.

$\mathrm{Xu}$, et al. [16] proposed the definition of ordinal inconsistency of an FPR as below.

Definition 6 [16]: "FPR $R=\left(r_{i j}\right)_{n \times n}$ is ordinally inconsistent if it has contradictory elements $r_{i j}, r_{i k}, r_{k j}$ for $i, j, k \in N$, $i \neq j \neq k \quad$ satisfying

$$
\begin{aligned}
& {\left[r_{i k}>0.5, r_{k j} \geq 0.5, r_{i j} \leq 0.5\right] \text { or }} \\
& {\left[r_{i k} \geq 0.5, r_{k j}>0.5, r_{i j} \leq 0.5\right] \text { or }} \\
& {\left[r_{i k}=0.5, r_{k j}=0.5, r_{i j} \neq 0.5\right] . ”}
\end{aligned}
$$

Definition 6 is equivalent to Definition 7.

Definition 7: "FPR $R=\left(r_{i j}\right)_{n \times n}$ is ordinally inconsistent if it has contradictory elements $r_{i j}, r_{i k}, r_{k j}$ for $i, j, k \in N$, $i \neq j \neq k \quad$ satisfying

$$
\begin{aligned}
& {\left[r_{i k}<0.5, r_{k j} \leq 0.5, r_{i j} \geq 0.5\right] \text { or }} \\
& {\left[r_{i k} \leq 0.5, r_{k j}<0.5, r_{i j} \geq 0.5\right] \text { or }} \\
& {\left[r_{i k}=0.5, r_{k j}=0.5, r_{i j} \neq 0.5\right] . "}
\end{aligned}
$$

The following result derives from Definitions 6 and 7:

Theorem 1: FPR $R=\left(r_{i j}\right)_{n \times n}$ is ordinally inconsistent, if and only if one the following cases is true.

Case a1: If $r_{i k} / r_{k i}>1, r_{k j} / r_{j k}>1$, then $r_{i j} / r_{j i}<1$;

Case a2: If $r_{i k} / r_{k i}>1, r_{k j} / r_{j k}>1$, then $r_{i j} / r_{j i}=1$;

Case a3: If $r_{i k} / r_{k i}>1, r_{k j} / r_{j k}=1$, then $r_{i j} / r_{j i}<1$; 
Case a4: If $r_{i k} / r_{k i}>1, r_{k j} / r_{j k}=1$, then $r_{i j} / r_{j i}=1$;

Case a5: If $r_{i k} / r_{k i}=1, r_{k j} / r_{j k}>1$, then $r_{i j} / r_{j i}<1$;

Case a6: If $r_{i k} / r_{k i}=1, r_{k j} / r_{j k}>1$, then $r_{i j} / r_{j i}=1$;

Case b1: If $r_{i k} / r_{k i}=1, r_{k j} / r_{j k}=1$, then $r_{i j} / r_{j i}<1$;

Case b2: If $r_{i k} / r_{k i}=1, r_{k j} / r_{j k}=1$, then $r_{i j} / r_{j i}>1$;

Case c1: If $r_{i k} / r_{k i}<1, r_{k j} / r_{j k}<1$, then $r_{i j} / r_{j i}>1$;

Case c2: If $r_{i k} / r_{k i}<1, r_{k j} / r_{j k}<1$, then $r_{i j} / r_{j i}=1$;

Case c3: If $r_{i k} / r_{k i}<1, r_{k j} / r_{j k}=1$, then $r_{i j} / r_{j i}>1$;

Case c4: If $r_{i k} / r_{k i}<1, r_{k j} / r_{j k}=1$, then $r_{i j} / r_{j i}=1$;

Case c5: If $r_{i k} / r_{k i}=1, r_{k j} / r_{j k}<1$, then $r_{i j} / r_{j i}>1$;

Case c6: If $r_{i k} / r_{k i}=1, r_{k j} / r_{j k}<1$, then $r_{i j} / r_{j i}=1$.

Proof. On the one hand, if $R$ is ordinally inconsistent, according to Definition 6 and Definition 7, it is easy to see that one of the above 14 possible cases is true. On the other hand, if one of the Cases a1-b1 is true, then it is $r_{i k} / r_{k i}, r_{k j} / r_{j k}, r_{j i} / r_{i j} \geq 1$ $(i \neq j \neq k) \quad$ (with at least one of them being $>1$ ), and therefore it would be $r_{i k}, r_{k j}, r_{j i} \geq 0.5$ (with at least one of them being >0.5). i.e., there would be a directed 3-cycles $x_{i} \succeq x_{k} \succeq x_{j} \succeq x_{i}$ (with at least one $\succ$ in the cycle) and the FPR would be ordinal inconsistent. If one of the Cases b2-c6 is true, then it is $r_{k i} / r_{i k}, r_{j k} / r_{k j}, r_{i j} / r_{j i} \geq 1(i \neq j \neq k)$ (with at least one of them being $>1$ ), which means that $r_{k i}, r_{j k}, r_{i j} \geq 0.5$ (with at least one of them being $>0.5$ ), i.e., there would be a direct 3-cycle $x_{i} \succeq x_{j} \succeq x_{k} \succeq x_{i}$ (with at least one $\succ$ in the cycle) and the FPR would be ordinal inconsistent.

Theorem 1 can in practice be used to find the contradictory elements of an FPR that lead to directed 3-cycles. It can also be used to propose a measurement of ordinal inconsistency of an FPR $R$ via the following Algorithm 2 designed to compute a symmetrical matrix $\Psi=\left(\psi_{i j}\right)_{n \times n}$ by detecting all possible combinations of three judgments and analyzing the ordinally inconsistent conditions.

\footnotetext{
Algorithm 2. Let $R=\left(r_{i j}\right)_{n \times n}$ be an FPR.

Set $\Psi=\operatorname{zero}(n)$

For all $i, j, k \quad(i \neq j \neq k \neq i)$ from 1 to $n$

If $\log \left(r_{i j} / r_{j i}\right) \cdot \log \left(r_{i k} / r_{k i}\right) \leq 0$ and $\log \left(r_{i j} / r_{j i}\right) \cdot \log \left(r_{k j} / r_{j k}\right)<0$, then$$
\psi_{i j}=\psi_{i j}+1
$$ 


$$
\begin{aligned}
& \text { If } \log \left(r_{i j} / r_{j i}\right) \cdot \log \left(r_{i k} / r_{k i}\right)<0 \text { and } \log \left(r_{i j} / r_{j i}\right) \cdot \log \left(r_{k j} / r_{j k}\right)=0 \text {, then } \\
& \psi_{i j}=\psi_{i j}+1 \\
& \text { If } \log \left(r_{i k} / r_{k i}\right)=0 \text { and } \log \left(r_{k j} / r_{j k}\right)=0 \text { and } \log \left(r_{i j} / r_{j i}\right) \neq 0 \text {, then } \\
& \quad \psi_{i j}=\psi_{i j}+1 \\
& \text { If } \log \left(r_{i k} / r_{k i}\right) \neq 0 \text { and } \log \left(r_{k j} / r_{j k}\right)=0 \text { and } \log \left(r_{i j} / r_{j i}\right)=0 \text {, then } \\
& \quad \psi_{i j}=\psi_{i j}+1 \\
& \text { If } \log \left(r_{i k} / r_{k i}\right)=0 \text { and } \log \left(r_{k j} / r_{j k}\right) \neq 0 \text { and } \log \left(r_{i j} / r_{j i}\right)=0 \text {, then } \\
& \psi_{i j}=\psi_{i j}+1 \\
& \text { If } \log \left(r_{i j} / r_{j i}\right)=0 \text { and } \log \left(r_{i k} / r_{k i}\right) \cdot \log \left(r_{k j} / r_{j k}\right)>0, \text { then } \\
& \psi_{i j}=\psi_{i j}+1
\end{aligned}
$$

End if

\section{End for}

Output $\Psi$

Notice that the smaller the value of $\psi_{i j}$, the more ordinally consistent the element $r_{i j}$ is. Thus, the largest value in $\Psi$ will correspond to the most ordinally inconsistent element in $R$, which would need to be rectified to improve the OC of $R$. Notice that in a directed 3-cycle with one inconsistent arc $x_{i} \rightarrow x_{j}$, the reverse is not in $G$. However, in the process of consistency improvement, due to the reciprocity property we need to change both the value of $r_{i j}$ and $r_{j i}$. If $r_{i j}$ is an ordinal inconsistent element which forms a directed 3-cycle, both $r_{i j}$ and $r_{j i}$ are in the same 3-cycle. Hence, in the process of improvement, we can consider any one of them.

We use the following example to demonstrate the usefulness of Algorithm 2.

\section{Example 2 (Example 1 continuation):}

By Algorithm 2, the matrix $\Psi=\left(\psi_{i j}\right)_{n \times n}$ is established:

$$
\Psi=\left[\begin{array}{llll}
0 & 1 & 1 & 2 \\
1 & 0 & 0 & 1 \\
1 & 0 & 0 & 1 \\
2 & 1 & 1 & 0
\end{array}\right]
$$

By observing the matrix $\Psi$, it is noticed that the element $r_{41}$ appears twice in all directed 3-cycles while the rest of values appear once. Therefore, by Theorem 4 there exist the following two directed 3-cycles:

$$
L=\left\{x_{1} \rightarrow x_{2} \rightarrow x_{4} \sim x_{1}, x_{1} \rightarrow x_{3} \rightarrow x_{4} \sim x_{1}\right\}
$$


Then, $r_{41}$ or $r_{14}$ are regarded as the most ordinally inconsistent elements.

\section{B. Overall $O C$}

The ordinal inconsistency of an FPR is measured by the number of directed 3-cycles present in its digraph. The following result provide a convenient relationship between the sum of the elements of matrix $\Psi$ and the number directed 3-cycles present in the digraph of an FPR.

Theorem 2: For FPR R, the sum of all elements in $\Psi=\left(\psi_{i j}\right)_{n \times n}$ is 6 times the number of directed 3-cycles in $G$.

Proof. The elements of $R$ in a directed 3-cycle in $G$ are contradictory and therefore by Algorithm 2 it is $\psi_{i j}=\psi_{j k}=\psi_{k i}=\psi_{j i}=\psi_{k j}=\psi_{i k}=1$. Thus, the sum of all elements in $\Psi=\left(\psi_{i j}\right)_{n \times n}$ is 6 times the number of directed 3-cycles in $\mathrm{G}$.

Theorem 2 justifies the below definition of overall OC of an FPR.

Definition 8: The overall OC of an FPR $R=\left(r_{i j}\right)_{n \times n}$ is:

$$
\psi(R)=\frac{1}{6} \sum_{i=1}^{n} \sum_{j=1}^{n} \psi_{i j}
$$

The following theorem is provided:

Theorem 3: An FPR $R=\left(r_{i j}\right)_{n \times n}$ is OC if and only if $\psi(R)=0$.

Proof: If $\mathrm{R}$ is $\mathrm{OC}$, then there is no directed 3 -cycles present in its digraph and consequently it will be $\psi(R)=0$. On the contrary, if $\psi(R)=0$ then it is $\psi_{i j}=0, \forall i, j \in N$ and therefore no element of $\mathrm{R}$ is present in a directed 3-cycle; consequently $R$ is OC.

The larger the value of $\psi(R)$, the less OC $\mathrm{R}$ is.

The FPR of Example 2 has an overall OC of $\psi\left(R_{1}\right)=2$, and it is not OC.

\section{An Algorithm For IMProving CONSISTENCIES}

When multiplicative and ordinally inconsistent judgments are detected, the next task is to remove them. In order to achieve this goal, in the following, an automatic procedure to guide the DM on how to revise inconsistent preference values are proposed.

In order to revise the inconsistent element $r_{i j} / r_{j i}$, all possible indirect judgments $\left(r_{i k} / r_{k i}\right) \cdot\left(r_{k j} / r_{j k}\right)$ are used to derive the following revised value of $r_{i j} / r_{j i}$

$$
\frac{r_{i j}^{\prime}}{r_{j i}^{\prime}}=\left(\prod_{k=1, k \neq i, k \neq j}^{n} \frac{r_{i k}}{r_{k i}} \cdot \frac{r_{k j}}{r_{j k}}\right)^{\frac{1}{n-2}}
$$

In FPRs, DMs tend to provide their preference information using the discrete scale $S_{[0.1,0.9]}=\{0.1,0.2,0.3,0.4,0.5,0.6$, 
$0.7,0.8,0.9\}$. However, the revised value $r_{i j}^{\prime}$ using Eq.(17) is generally not in this scale. Thus, to make the revised values in this scale, values for $r_{i j}^{\prime} / r_{j i}^{\prime}$ via Eq.(17) will be matched with the Table III closet to ratio value in the $S_{[0.1,0.9]}$.

TABLE III. THE RATIOS CORRESPONDING TO THE VALUE IN THE SCALE $\mathrm{S}_{[0.1,0.9]}$

\begin{tabular}{c|c|c|c|c|c|c|c|c|c}
\hline$\frac{r_{i j}}{r_{j i}}$ & $\frac{0.1}{0.9}$ & $\frac{0.2}{0.8}$ & $\frac{0.3}{0.7}$ & $\frac{0.4}{0.6}$ & $\frac{0.5}{0.5}$ & $\frac{0.6}{0.4}$ & $\frac{0.7}{0.3}$ & $\frac{0.8}{0.2}$ & $\frac{0.9}{0.1}$ \\
\hline Ratio & 0.1111 & 0.25 & 0.4286 & 0.6667 & 1 & 1.5 & 2.3333 & 4 & 9 \\
\hline
\end{tabular}

In particular, when the obtained value $r_{i j}^{\prime} / r_{j i}^{\prime}$ is greater than 9 , then the following will be set $r_{i j}^{\prime}=0.9$; while for a $r_{i j}^{\prime} / r_{j i}^{\prime}$ lower than 0.11111 , it will be set $r_{i j}^{\prime}=0.1$. Meanwhile, $r_{i j}^{\prime} / r_{j i}^{\prime}$ is close to 1 it will be $r_{i j}^{\prime}=0.6$ when $r_{i j}^{\prime} / r_{j i}^{\prime}>1$, and $r_{i j}^{\prime}=0.4$ when $r_{i j}^{\prime} / r_{j i}^{\prime}<1$

Based on Algorithms 1 and 2, the automatic approach to identify and repair inconsistent judgments is detailed in Algorithm 3 and depicted in Fig. 2.

Algorithm 3. Let $R=\left(r_{i j}\right)_{n \times n}$ be an FPR and let $t$ be the number of iterations.

Step 1. Let $R^{(t)}=\left(r_{i j}^{(t)}\right)_{n \times n}=\left(r_{i j}\right)_{n \times n}$ and $t=0$.

Step 2. Calculate the priority vector $w^{(t)}=\left(w_{1}^{(t)}, w_{2}^{(t)}, \cdots, w_{n}^{(t)}\right)^{T}$ of $R^{(t)}$ by Eq.(8).

Step 3. Construct the matrix $\Psi^{(t)}=\left(\psi_{i j}^{(t)}\right)_{n \times n}$ by Algorithm 2.

Step 4. Compute $G C I\left(R^{(t)}\right)$ by Eq.(7) and $\psi\left(R^{(t)}\right)$ by Eq.(16).

Step 5. $R^{(t)}$ can be classified into the following four types.

Step 5A: If $R^{(t)}$ is both multiplicative and ordinally inconsistent $\left(G C I\left(R^{(t)}\right)>\overline{G C I(R)}, \psi\left(R^{(t)}\right) \neq 0\right)$, go to Step 6A.

Step 5B: If $R^{(t)}$ is MC but ordinally inconsistent $\left(G C I\left(R^{(t)}\right) \leq \overline{G C I(R)}, \psi\left(R^{(t)}\right) \neq 0\right)$, go to Step 6B.

Step 5C: If $R^{(t)}$ is OC but multiplicative inconsistent $\left(\psi\left(R^{(t)}\right)=0, G C I\left(R^{(t)}\right)>\overline{G C I(R)}\right)$, go to Step 6C.

Step 5D: If $R^{(t)}$ is both MC and OC, that is $G C I\left(R^{(t)}\right) \leq \overline{G C I(R)}, \psi\left(R^{(t)}\right)=0$, then go to Step 9.

Step 6. The treatments of inconsistencies.

Step 6A: Following Step 5A:

(1) According to Algorithm 1, establish matrix $\Theta^{(t)}=\left(\theta_{i j}^{(t)}\right)_{n \times n}$.

(2) Find $\psi_{i_{o} j_{o}}^{(t)}=\max _{i, j}\left\{\psi_{i j}^{(t)}\right\}$ in matrix $\Psi^{(t)}=\left(\psi_{i j}^{(t)}\right)_{n \times n}$ and $\theta_{i_{m} j_{m}}^{(t)}=\max _{i, j}\left\{\theta_{i j}^{(t)}\right\}$ in matrix $\Theta^{(t)}=\left(\theta_{i j}^{(t)}\right)_{n \times n}$.

(3) If the corresponding $r_{i_{m} j_{m}}^{(t)}$ and $r_{i_{o} j_{o}}^{(t)}$ are the same element (i.e., $i_{m}=i_{o}, j_{m}=j_{o}$ ), then it is regarded as the most inconsistent element to be adjusted. Otherwise, we automatically select the corresponding $r_{i_{o} j_{o}}^{(t)}$ as the most inconsistent element to be adjusted. 
(4) Compute the value of $r_{i j}^{\prime(t)} / r_{j i}^{\prime(t)}$ using Eq.(17) and identify the closest ratio to $r_{i j}^{\prime(t)} / r_{j i}^{\prime(t)}$ in Table III. Then find the revised value $r_{i j}^{\prime(t)}$ and go to next step.

Step 6B: Following Step 5B:

(1) Find $\psi_{i_{o} j_{o}}^{(t)}=\max _{i, j}\left\{\psi_{i j}^{(t)}\right\}$ in matrix $\Psi^{(t)}=\left(\psi_{i j}^{(t)}\right)_{n \times n}$, then the corresponding $r_{i_{o} j_{o}}^{(t)}$ in $R^{(t)}$ is regarded as the most ordinally inconsistent element. If there are two or more most ordinally inconsistent elements, choose any one to be the most ordinally inconsistent one.

(2) Compute the value of $r_{i j}^{\prime(t)} / r_{j i}^{\prime(t)}$ using Eq.(17) and identify the closest ratio to $r_{i j}^{\prime(t)} / r_{j i}^{\prime(t)}$ in Table III. Then find the revised value $r_{i j}^{\prime(t)}$ and go to next step.

(3) If the value of $r_{i j}^{\prime(t)}$ satisfies $\log \left(r_{i j}^{(t)} / r_{j i}^{(t)}\right) \cdot \log \left(r_{i j}^{\prime(t)} / r_{j i}^{\prime(t)}\right)<0$, go to next step. Otherwise, we set $r_{i j}^{\prime(t)}=1-r_{i j}^{(t)}$, $r_{j i}^{(t)}=r_{i j}^{(t)}$

Step 6C: Following Step 5C:

(1) According to Algorithm 1, establish matrix $\Theta^{(t)}=\left(\theta_{i j}^{(t)}\right)_{n \times n}$.

(2) Find $\theta_{i_{m} j_{m}}^{(t)}=\max _{i, j}\left\{\theta_{i j}^{(t)}\right\}$ in matrix $\Theta^{(t)}=\left(\theta_{i j}^{(t)}\right)_{n \times n}$, then the corresponding $r_{i_{m} j_{m}}^{(t)}$ in $R^{(t)}$ is regarded as the most multiplicative inconsistent element.

(3) Compute the value of $r_{i j}^{\prime(t)} / r_{j i}^{\prime(t)}$ using Eq.(17) and identify the closest ratio value from Table III. Then find the revised value $r_{i j}^{(t)}$.

(4) If the value of $r_{i j}^{\prime(t)}$ satisfies $\log \left(r_{i j}^{(t)} / r_{j i}^{(t)}\right) \cdot \log \left(r_{i j}^{\prime(t)} / r_{j i}^{(t)}\right)>0$, go to Step 7. Otherwise, go back to (2) to search the second largest value in matrix $\Theta^{(t)}=\left(\theta_{i j}^{(t)}\right)_{n \times n}$ to improve.

Step 7. New improved FPR $R^{(t+1)}=\left(r_{i j}^{(t+1)}\right)_{n \times n}$, where

$$
\left(r_{i j}^{(t+1)}, r_{j i}^{(t+1)}\right)= \begin{cases}\left(r_{i j}^{(t)}, 1-r_{i j}^{(t)}\right), & \text { if } r_{i j}^{(t)} \text { is the inconsistent element } \\ \left(r_{i j}^{(t)}, r_{j i}^{(t)}\right), & \text { otherwise }\end{cases}
$$

Step 8. Let $t=t+1$, then go back to Step 2 .

Step 9. Output $t, R^{(t)}, \psi\left(R^{(t)}\right)$ and $G C I\left(R^{(t)}\right)$.

Step 10. End.

Remark 4: In Step 6A, after finding the largest value $\psi_{i_{o} j_{o}}^{(t)}$ and $\theta_{i_{m} j_{m}}^{(t)}$, the most multiplicative inconsistent element $r_{i_{m} j_{m}}^{(t)}$ and the most ordinally inconsistent element $r_{i_{o} j_{o}}^{(t)}$ in $R^{(t)}$ are identified. If $r_{i_{m} j_{m}}^{(t)}$ and $r_{i_{o} j_{o}}^{(t)}$ are the same $(i, j)$ element in $R$, 
we naturally adopt the above improved method; Otherwise, priority is given to revise the ordinally inconsistent element because it is considered the minimum requirement for the judgement information not to be illogical. This explains why in Algorithm 3, the ordinally inconsistent element $r_{i_{o} j_{o}}^{(t)}$ is selected to be the rectified element when both inconsistencies happen in different fuzzy preference value positions.

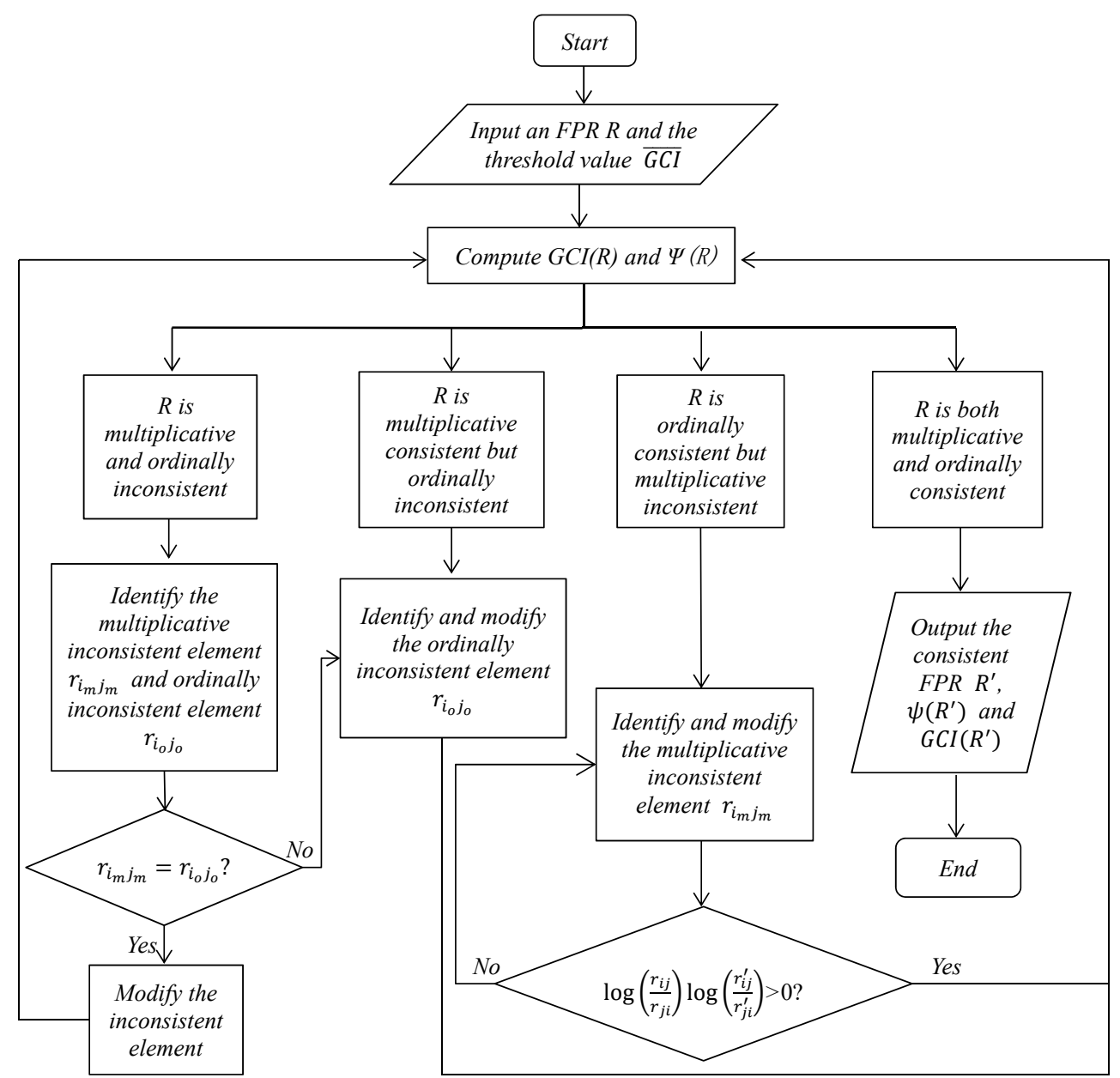

Fig. 2. The process of identifying and repairing inconsistent judgments in Algorithm 3

Remark 5: In Algorithm 3, we identify the most inconsistent element (i.e. search the maximum value in the matrix $\Psi^{(t)}=\left(\psi_{i j}^{(t)}\right)_{n \times n}$ and $\left.\Theta^{(t)}=\left(\theta_{i j}^{(t)}\right)_{n \times n}\right)$. If there exist more than one inconsistent element in $R^{(t)}$ with maximum value, then the inconsistent element with value closer to 0.5 is selected. If more than one value results again, then any of them is selected (randomly, for example) as the inconsistent element to adjust.

Remark 6: In Step 6B, when the FPR is ordinally inconsistent but MC, the revised values should reverse the direction of the ordinally inconsistent elements. If the revised values do not satisfy this requirement, i.e., $\log \left(r_{i j}^{(t)} / r_{j i}^{(t)}\right) \cdot \log \left(r_{i j}^{\prime(t)} / r_{j i}^{(t)}\right)<0$, then we just reverse its direction by changing their original values. If there are two or more 
ordinally inconsistent elements, we choose any one to reverse it, this does not affect the final result. This is also verified in our Monte Carlo simulation experiments. In Step 6C, the FPR is the OC but multiplicative inconsistent (i.e., $\psi\left(R^{(t)}\right)=0$ and $\left.G C I\left(R^{(t)}\right)>\overline{G C I(R)}\right)$. Thus, the revised value $r_{i j}^{\prime(t)}$ should not reverse the direction of the original value $r_{i j}^{(t)}$. In order to achieve this goal, the constraint $\log \left(r_{i j}^{(t)} / r_{j i}^{(t)}\right) \cdot \log \left(r_{i j}^{(t)} / r_{j i}^{(t)}\right)>0$ is imposed, i.e., if $\quad r_{i j}^{(t)}>0.5\left(\right.$ i.e., $\left.\quad r_{i j}^{(t)} / r_{j i}^{(t)}>1\right)$, then the adjusted value $r_{i j}^{\prime(t)}$ also should be larger than 0.5 , i.e., $r_{i j}^{\prime(t)} / r_{j i}^{(t)}>1$. Similarly, if $r_{i j}^{(t)}<0.5,\left(\right.$ i.e.,,$\left.r_{i j}^{(t)} / r_{j i}^{(t)}<1\right)$, then the adjusted value $r_{i j}^{\prime(t)}$ should be lower than 0.5 , i.e., $r_{i j}^{\prime(t)} / r_{j i}^{\prime(t)}<1$. Therefore, the revised element should satisfy $\log \left(r_{i j}^{(t)} / r_{j i}^{(t)}\right) \cdot \log \left(r_{i j}^{(t)} / r_{j i}^{(t)}\right)>0$. Otherwise, the second largest value in matrix $\Theta^{(t)}=\left(\theta_{i j}^{(t)}\right)_{n \times n}$ is chosen to be improved. This will guarantee the construction of an FPR with acceptable MC and OC.

\section{IlluSTRATIVE EXAMPLES, COMPARATIVE ANALYSES AND DISCUSSIONS}

This section includes two subsections. The first one offers some illustrative examples, and comparisons with the previous methods in literature. The second one provides some simulation experiments to additionally support the effectiveness of the proposed methods.

\section{A. Illustrative Examples and Comparative Analyses}

In this section, we will offer four examples, one for each type of inconsistency situation of Algorithm 3 (Fig. 2), to demonstrate the usefulness and feasibility of our developed methods.

- Example 3 is the continuation of Example 1, which has been studied in $\mathrm{Xu}$, et al. [15], and corresponds to the case of an FPR that is both multiplicative and ordinally inconsistent, with same ordinal and multiplicative inconsistent elements.

- Example 4 was investigated by $\mathrm{Xia}$, et al. [13], and corresponds to an FPR that is OC but multiplicative inconsistency.

- Example 5 was examined by $\mathrm{Xu}$, et al. [29]. It corresponds to an FPR that is ordinally inconsistent but with acceptable MC.

- Example 6 is from $\mathrm{Xu}$ and Cai [35], and it covers the case of an FPR that is both ordinally and multiplicative inconsistent, with different ordinal and multiplicative inconsistent elements.

Comparisons with the previous approaches in the literature are also provided for each example.

Example 3 (Example 1 continuation): In this example, we will adjust the multiplicative and ordinal inconsistencies of an FPR which has the same ordinal and multiplicative inconsistent elements.

Step 1. Let $R_{1}^{(0)}=\left(r_{i j}^{(0)}\right)_{4 \times 4}=\left(r_{i j}\right)_{4 \times 4}, t=0$, and $\overline{G C I\left(R_{1}\right)}=0.3526$. 
Step 2. By Eq.(8), $w^{(0)}=(0.4697,0.2428,0.1619,0.1255)^{T}$. By Eq.(7), it is $G C I\left(R_{1}^{(0)}\right)=1.4581>\overline{G C I\left(R_{1}\right)}$. Thus, $R_{1}^{(0)}$ is multiplicative inconsistent.

Step 3. In Example 2, it was obtained the following the matrix $\Psi^{(0)}=\left(\psi_{i j}^{(0)}\right)_{4 \times 4}$ :

$$
\Psi^{(0)}=\left[\begin{array}{llll}
0 & 1 & 1 & 2 \\
1 & 0 & 0 & 1 \\
1 & 0 & 0 & 1 \\
2 & 1 & 1 & 0
\end{array}\right]
$$

Step 4. By Eq.(16), $\psi\left(R_{1}^{(0)}\right)=2 \neq 0$. It means that $R_{1}^{(0)}$ is also ordinally inconsistent.

Step 5. According to Algorithm 1, due to different definitions of $\delta$ and $\theta_{i j}$, we may get many forms of $\Theta^{(0)}=\left(\theta_{i j}^{(0)}\right)_{n \times n}$ which are shown in Table I. For the convenience of explanation, we use the following matrix established by Eq.(9) and Eq.(11) as an example.

$$
\Theta^{(0)}=\left[\begin{array}{cccc}
0 & 1.3195 & 2.2640 & 2.6391 \\
1.3195 & 0 & 0.9445 & 1.3195 \\
2.2640 & 0.9445 & 0 & 2.2640 \\
2.6391 & 1.3195 & 2.2640 & 0
\end{array}\right]
$$

Step 6. Observing the matrix $\Psi^{(0)}$, the largest value in $\Psi^{(0)}$ is $\psi_{14}^{(0)}=\psi_{41}^{(0)}=2$. Meanwhile, the largest value in the matrix $\Theta^{(0)}$ is $\theta_{14}^{(0)}=\theta_{41}^{(0)}=2.6391$. Therefore, $\quad r_{14}^{(0)}$ is the most inconsistent element.

Step 7. By Eq.(17), the new value of $r_{14}^{\prime(0)} / r_{41}^{\prime(0)}=14>9$ is derived, and therefore it is set $r_{14}^{\prime(0)}=0.9$.

Step 8. According to Eq.(18), the following improved FPR $R_{1}^{(1)}$ is derived:

$$
R_{1}^{(1)}=\left[\begin{array}{llll}
0.5 & 0.7 & 0.9 & 0.9 \\
0.3 & 0.5 & 0.6 & 0.7 \\
0.1 & 0.4 & 0.5 & 0.8 \\
0.1 & 0.3 & 0.2 & 0.5
\end{array}\right]
$$

Step 9. By Eq.(8), $w^{(1)}=(0.6303,0.1881,0.1254,0.0562)^{T}$, and the ranking of alternatives is $x_{1} \succ x_{2} \succ x_{3} \succ x_{4}$.

Step 10. By Algorithm 2, the matrix $\Psi^{(1)}=\left(\psi_{i j}^{(1)}\right)_{4 \times 4}$ is:

$$
\Psi^{(1)}=\left[\begin{array}{llll}
0 & 0 & 0 & 0 \\
0 & 0 & 0 & 0 \\
0 & 0 & 0 & 0 \\
0 & 0 & 0 & 0
\end{array}\right]
$$

Step 11. By Eq.(7), $G C I\left(R_{1}^{(1)}\right)=0.3299<\overline{G C I\left(R_{1}\right)}$. By Eq.(16), $\psi\left(R_{1}^{(1)}\right)=0$. Thus, $R_{1}^{(1)}$ is OC and of acceptable MC.

Through the proposed consistency improvement process, an FPR $R_{1}^{(1)}$ with OC and acceptable MC is eventually obtained. Furthermore, the ranking of the alternatives that is derived from $R_{1}^{(1)}$ coincides with the initial ranking result. 
In this example, because there exist equal preferences ( i.e. $r_{14}=r_{41}=0.5$ ), Ma, et al. [30]'s method fails to improve consistency. Xu, et al. [15]'s method can only remove the ordinal inconsistency because MC is not considered. Furthermore, $\mathrm{Xu}$, et al. [15] derived the following adjusted OC consistent FPR:

$$
R_{1}=\left[\begin{array}{llll}
0.5 & 0.7 & 0.9 & 0.6 \\
0.3 & 0.5 & 0.6 & 0.7 \\
0.1 & 0.4 & 0.5 & 0.8 \\
0.4 & 0.3 & 0.2 & 0.5
\end{array}\right]
$$

When Eq.(7) is applied, it is observed that $G C I\left(R_{1}\right)=1.1288>\overline{G C I\left(R_{1}\right)}$, i.e., $R_{1}$ is not of acceptable MC. It is evident that the proposed method in this paper is more efficient than existing ones, because it can improve the OC and MC simultaneously in a single model.

Example 4: Suppose the following an FPR on four alternatives $x_{1}, x_{2}, x_{3}$ and $x_{4}$ (adapted from Xia, et al. [13]):

$$
R_{2}=\left[\begin{array}{llll}
0.5 & 0.4 & 0.7 & 0.3 \\
0.6 & 0.5 & 0.6 & 0.8 \\
0.3 & 0.4 & 0.5 & 0.3 \\
0.7 & 0.2 & 0.7 & 0.5
\end{array}\right]
$$

In this example, we will deal with an ordinal consistent but multiplicative inconsistent FPR.

Step 1. Let $R_{2}^{(0)}=\left(r_{i j}^{(0)}\right)_{4 \times 4}=\left(r_{i j}\right)_{4 \times 4}, t=0$, and $\overline{G C I\left(R_{2}\right)}=0.3526$.

Step 2. By Eq.(8), the priority vector of $R_{2}^{(0)}$ is $w^{(0)}=(0.2098,0.4021,0.1373,0.2508)^{T}$.

Step 3. By Algorithm 2, the following matrix $\Psi=\left(\psi_{i j}\right)_{n \times n}$ was obtained:

$$
\Psi^{(0)}=\left[\begin{array}{llll}
0 & 0 & 0 & 0 \\
0 & 0 & 0 & 0 \\
0 & 0 & 0 & 0 \\
0 & 0 & 0 & 0
\end{array}\right]
$$

Step 4. By Eq.(7), $G C I\left(R_{2}^{(0)}\right)=0.6767>\overline{G C I\left(R_{2}\right)}$. By Eq.(16), the value of $\psi\left(R_{2}^{(0)}\right)$ is equal to 0 . Thus, $R_{2}^{(0)}$ is OC but multiplicative inconsistent. Thus, only MC of $R_{2}^{(0)}$ is to be improved.

Step 5. According to Eq.(9) and Eq.(11), the matrix $\Theta^{(0)}=\left(\theta_{i j}^{(0)}\right)_{n \times n}$ :

$$
\Theta^{(0)}=\left[\begin{array}{cccc}
0 & 1.3377 & 0.8473 & 1.3377 \\
1.3377 & 0 & 1.3377 & 1.8281 \\
0.8473 & 1.3377 & 0 & 1.3377 \\
1.3377 & 1.8281 & 1.3377 & 0
\end{array}\right]
$$

Step 6. The largest value in $\Theta^{(0)}$ is $\theta_{24}^{(0)}=\theta_{42}^{(0)}=1.8281$. Thus, $r_{24}^{(0)}$ in $R_{2}^{(0)}$ is the most multiplicative inconsistent element that requires to be improved.

Step 7. By Eq.(17), $r_{24}^{\prime(0)} / r_{42}^{\prime(0)}=0.6429$ and it is closet to $0.4 / 0.6$ in Table III. Then $r_{24}^{\prime(0)}=0.4$. 
Step 8. However, it is $\log \left(r_{24}^{(0)} / r_{42}^{(0)}\right) \cdot \log \left(r_{24}^{\prime(0)} / r_{42}^{\prime(0)}\right)<0$, which means that the new value $r_{24}^{\prime(0)}$ changes the original order.

Thus, the second largest value in matrix $\Theta^{(0)}$ is to be found and improved.

Step 9. The second largest value in matrix $\Theta^{(0)}$ is $\theta_{12}^{(0)}=\theta_{21}^{(0)}=\theta_{14}^{(0)}=\theta_{41}^{(0)}=\theta_{23}^{(0)}=\theta_{32}^{(0)}=\theta_{34}^{(0)}=\theta_{43}^{(0)}=1.3377$. According to Remark 5, any of the values $r_{12}^{(0)}, r_{14}^{(0)}, r_{23}^{(0)}, r_{34}^{(0)}$ can be selected as the most multiplicative inconsistent element. Here, $r_{23}^{(0)}$ is selected.

Step 10. By Eq.(17), we get the value of $r_{23}^{\prime(0)} / r_{32}^{\prime(0)}=5.7155$, which is closest to $0.8 / 0.2$ in Table III. This means that $r_{23}^{\prime(0)}=0.8$

Step 11. Because the values of $r_{23}^{(0)}$ and $r_{23}^{\prime(0)}$ satisfy $\log \left(r_{23}^{(0)} / r_{32}^{(0)}\right) \cdot \log \left(r_{23}^{\prime(0)} / r_{32}^{\prime(0)}\right)>0$, the value of $r_{23}^{\prime(0)}$ does not modify the OC.

Step 12. By Eq.(18), the following improved FPR $R_{2}^{(1)}$ is obtained:

$$
R_{2}^{(1)}=\left[\begin{array}{llll}
0.5 & 0.4 & 0.7 & 0.3 \\
0.6 & 0.5 & 0.8 & 0.8 \\
0.3 & 0.2 & 0.5 & 0.3 \\
0.7 & 0.2 & 0.7 & 0.5
\end{array}\right]
$$

Step 13. By Eq.(8), it is $w^{(1)}=(0.1939,0.4750,0.0993,0.2318)^{T}$.

Step 14. By Eq.(7), because $G C I\left(R_{2}^{(1)}\right)=0.3996>\overline{G C I\left(R_{2}\right)}$, then $R_{2}^{(1)}$ is still of unacceptable MC. Therefore, the MC needs further improvement.

Step 15. According to Eq.(9) and Eq.(11), the new matrix $\Theta^{(1)}=\left(\theta_{i j}^{(1)}\right)_{n \times n}$ is:

$$
\Theta^{(1)}=\left[\begin{array}{cccc}
0 & 0.9808 & 0.4904 & 1.3377 \\
0.9808 & 0 & 0.4904 & 1.3377 \\
0.4904 & 0.4904 & 0 & 0.8473 \\
1.3377 & 1.3377 & 0.8473 & 0
\end{array}\right]
$$

Step 16. The maximum value in the matrix $\Theta^{(1)}$ is $\theta_{14}^{(1)}=\theta_{41}^{(1)}=\theta_{24}^{(1)}=\theta_{42}^{(1)}=1.3377$. As per Remark 5 , $r_{24}^{(1)}$ in $R_{2}^{(1)}$ is selected as the multiplicative inconsistent element.

Step 17. By Eq.(17), it is $r_{24}^{\prime(1)} / r_{42}^{\prime(1)}=1.0498>1$, which leads to $r_{24}^{\prime(1)}=0.6$.

Step 18. Because the values of $r_{24}^{(1)}$ and $r_{24}^{\prime(1)}$ satisfy $\log \left(r_{24}^{(1)} / r_{42}^{(1)}\right) \cdot \log \left(r_{24}^{\prime(1)} / r_{42}^{\prime(1)}\right)>0, \quad r_{24}^{\prime(1)}$ does not change the OC.

Step 19. By Eq.(18), the new improved FPR $R_{2}^{(2)}$ is: 


$$
R_{2}^{(2)}=\left[\begin{array}{llll}
0.5 & 0.4 & 0.7 & 0.3 \\
0.6 & 0.5 & 0.8 & 0.6 \\
0.3 & 0.2 & 0.5 & 0.3 \\
0.7 & 0.4 & 0.7 & 0.5
\end{array}\right]
$$

Step 20. By Eq.(8), $w^{(2)}=(0.2017,0.3867,0.1034,0.3082)^{T}$, i.e., $\quad x_{2} \succ x_{4} \succ x_{1} \succ x_{3}$.

Step 21. By Eq.(7), $G C I\left(R_{2}^{(2)}\right)=0.1226<\overline{G C I\left(R_{2}\right)}$, which means that $R_{2}^{(2)}$ is of acceptable MC.

Finally, $R_{2}^{(2)}$ is both MC and OC. Furthermore, the derived ranking of alternatives $x_{2} \succ x_{4} \succ x_{1} \succ x_{3}$ coincides with the initial ranking.

Xia, et al. [13]'s method requires 4 iterations to derive the following relation of acceptable MC [for $\overline{G C I\left(R_{2}\right)}=0.3526$, $\theta=0.1 \quad(\theta$ is a parameter in Xia, et al. [13]'s method)]:

$$
R_{2}^{(4)}=\left[\begin{array}{llll}
0.5000 & 0.3799 & 0.6685 & 0.3503 \\
0.6201 & 0.5000 & 0.6536 & 0.7450 \\
0.3315 & 0.3464 & 0.5000 & 0.3181 \\
0.6497 & 0.2550 & 0.6819 & 0.5000
\end{array}\right], \quad G C I\left(R_{2}^{(4)}\right)=0.2915<\overline{G C I\left(R_{2}\right)}
$$

This shows that Xia, et al. [13]'s approach is computationally more expensive than the present method, in addition to modifying all the original preference values except those on the main diagonal. However, the proposed method requires the modification of only 4 original preference values; the improved FPR has a lower $G C I\left(R_{2}^{(2)}\right)$ of 0.1226 , and therefore it is more MC than the improved FPR derived by Xia, et al. [13].

Example 5: Assume that a DM provides the following preferences over a set of six alternatives (adapted from [29]):

$$
R_{3}=\left[\begin{array}{llllll}
0.5 & 0.7 & 0.3 & 0.7 & 0.3 & 0.7 \\
0.3 & 0.5 & 0.6 & 0.5 & 0.2 & 0.5 \\
0.7 & 0.4 & 0.5 & 0.8 & 0.5 & 0.8 \\
0.3 & 0.5 & 0.2 & 0.5 & 0.2 & 0.5 \\
0.7 & 0.8 & 0.5 & 0.8 & 0.5 & 0.8 \\
0.3 & 0.5 & 0.2 & 0.5 & 0.2 & 0.5
\end{array}\right]
$$

In this example, an FPR that is ordinally inconsistent but with acceptable MC needs to be repaired.

Step 1. Let $R_{3}^{(0)}=\left(r_{i j}^{(0)}\right)_{6 \times 6}=\left(r_{i j}\right)_{6 \times 6}, t=0$, and the Table I threshold value $\overline{\operatorname{GCI}\left(R_{3}\right)}=0.37$.

Step 2. By Eq.(8), the priority vector of $R_{3}^{(0)}$ is $w^{(0)}=(0.1646,0.1054,0.2443,0.0782,0.3293,0.0782)^{T}$. By Eq.(7), $G C I\left(R_{3}^{(0)}\right)=0.2419<\overline{G C I\left(R_{3}\right)}$. Thus, $R_{3}^{(0)}$ is acceptable MC.

Step 3. According to Algorithm 2, the matrix $\Psi^{(0)}=\left(\psi_{i j}^{(0)}\right)_{6 \times 6}$ : 


$$
\Psi^{(0)}=\left[\begin{array}{llllll}
0 & 1 & 1 & 0 & 0 & 0 \\
1 & 0 & 4 & 1 & 1 & 1 \\
1 & 4 & 0 & 1 & 1 & 1 \\
0 & 1 & 1 & 0 & 0 & 0 \\
0 & 1 & 1 & 0 & 0 & 0 \\
0 & 1 & 1 & 0 & 0 & 0
\end{array}\right]
$$

Step 4. By Eq.(16), $\psi\left(R_{3}^{(0)}\right)=4$. It means that $R_{3}^{(0)}$ is ordinally inconsistent.

Step 5. In $\Psi^{(0)}$, the largest value is $\psi_{23}^{(0)}=\psi_{32}^{(0)}=4$, then $r_{23}^{(0)}$ is the most ordinally inconsistent element.

Step 6. By Eq.(17), we have: $r_{23}^{\prime(0)} / r_{32}^{(0)}=0.2315$ and it is closest to $0.2 / 0.8$ in Table III. Then $r_{23}^{\prime(0)}=0.2$.

Step 7. According to Eq.(18), the following improved FPR $R_{3}^{(1)}$ is derived:

$$
R_{3}^{(1)}=\left[\begin{array}{llllll}
0.5 & 0.7 & 0.3 & 0.7 & 0.3 & 0.7 \\
0.3 & 0.5 & 0.2 & 0.5 & 0.2 & 0.5 \\
0.7 & 0.8 & 0.5 & 0.8 & 0.5 & 0.8 \\
0.3 & 0.5 & 0.2 & 0.5 & 0.2 & 0.5 \\
0.7 & 0.8 & 0.5 & 0.8 & 0.5 & 0.8 \\
0.3 & 0.5 & 0.2 & 0.5 & 0.2 & 0.5
\end{array}\right]
$$

Step 8. By Eq.(8), it is $w^{(1)}=(0.1556,0.0739,0.3113,0.0739,0.3113,0.0739)^{T}$.

Step 9. By Algorithm 2, the matrix $\Psi^{(1)}=\left(\psi_{i j}^{(1)}\right)_{6 \times 6}$ is:

$$
\Psi^{(1)}=\left[\begin{array}{llllll}
0 & 0 & 0 & 0 & 0 & 0 \\
0 & 0 & 0 & 0 & 0 & 0 \\
0 & 0 & 0 & 0 & 0 & 0 \\
0 & 0 & 0 & 0 & 0 & 0 \\
0 & 0 & 0 & 0 & 0 & 0 \\
0 & 0 & 0 & 0 & 0 & 0
\end{array}\right]
$$

Step 10. By Eq.(7), $G C I\left(R_{3}^{(1)}\right)=0.0095<\overline{G C I\left(R_{3}\right)}$. By Eq.(16), $\psi\left(R_{3}^{(1)}\right)=0$. Thus, $R_{3}^{(1)}$ is now both MC and OC.

The ranking of alternatives will be: $x_{3} \sim x_{5} \succ x_{1} \succ x_{2} \sim x_{4} \sim x_{6}$.

This example was investigated by $\mathrm{Xu}$, et al. [29], where they derived the following improved FPR:

$$
R_{3}^{\prime}=\left[\begin{array}{llllll}
0.5 & 0.7 & 0.3 & 0.7 & 0.3 & 0.7 \\
0.3 & 0.5 & 0.4 & 0.5 & 0.2 & 0.5 \\
0.7 & 0.6 & 0.5 & 0.8 & 0.5 & 0.8 \\
0.3 & 0.5 & 0.2 & 0.5 & 0.2 & 0.5 \\
0.7 & 0.8 & 0.5 & 0.8 & 0.5 & 0.8 \\
0.3 & 0.5 & 0.2 & 0.5 & 0.2 & 0.5
\end{array}\right]
$$

By Eq. (7), the weighting vector of $R_{3}^{\prime}$ is $w=(0.1611,0.0901,0.2736,0.0765,0.3222,0.0765)^{T}$ and it has $G C I\left(R_{3}^{\prime}\right)=$ 0.0837. Then the ranking of alternatives for $R_{3}^{\prime}$ would be: $x_{5} \succ x_{3} \succ x_{1} \succ x_{2} \succ x_{4} \sim x_{6}$. As we can see in $R_{3}$, the original 
indifference between pairs of alternatives (for example between $x_{5}$ and $x_{3}: x_{5} \sim x_{3}, r_{35}=0.5$ ) is kept by the proposed method but not by $\mathrm{Xu}$, et al. [29]'s method. Again, this example shows that the proposed method is able to improve the OC and the MC simultaneously. After adjusting the inconsistent elements, the inconsistent elements are eliminated, and the degree of GCI is greatly improved from 0.2419 to 0.0095 .

Example 6: Suppose a DM gives an FPR on the set of 6 alternative as follows (adapted from [35]):

$$
R_{4}=\left[\begin{array}{llllll}
0.5 & 0.3 & 0.3 & 0.7 & 0.8 & 0.5 \\
0.7 & 0.5 & 0.2 & 0.7 & 0.8 & 0.6 \\
0.7 & 0.8 & 0.5 & 0.7 & 0.7 & 0.8 \\
0.3 & 0.3 & 0.3 & 0.5 & 0.9 & 0.7 \\
0.2 & 0.2 & 0.3 & 0.1 & 0.5 & 0.4 \\
0.5 & 0.4 & 0.2 & 0.3 & 0.6 & 0.5
\end{array}\right]
$$

In this example, we will rectify the multiplicative and ordinal inconsistencies of an FPR that has different ordinal and multiplicative inconsistent elements.

Step 1. Let $R_{4}^{(0)}=\left(r_{i j}^{(0)}\right)_{6 \times 6}=\left(r_{i j}\right)_{6 \times 6}, t=0$, and Table I value $\overline{G C I\left(R_{4}\right)}=0.37$

Step 2. By Eq.(8), the priority vector of $R_{4}^{(0)}$ is $w^{(0)}=(0.1548,0.2007,0.3430,0.1538,0.0501,0.0975)^{T}$. By Eq.(7), $G C I\left(R_{4}^{(0)}\right)=0.5051>\overline{G C I\left(R_{4}\right)}$, which means that is not of acceptable MC.

Step 3. According to Algorithm 2, the matrix $\Psi^{(0)}=\left(\psi_{i j}^{(0)}\right)_{6 \times 6}$ :

$$
\Psi^{(0)}=\left[\begin{array}{llllll}
0 & 0 & 0 & 1 & 0 & 1 \\
0 & 0 & 0 & 0 & 0 & 0 \\
0 & 0 & 0 & 0 & 0 & 0 \\
1 & 0 & 0 & 0 & 0 & 1 \\
0 & 0 & 0 & 0 & 0 & 0 \\
1 & 0 & 0 & 1 & 0 & 0
\end{array}\right]
$$

Step 4. By Eq.(16), $\psi\left(R_{4}^{(0)}\right)=1$. It means that $R_{4}^{(0)}$ is also ordinally inconsistent.

Step 5. By Eq.(9) and Eq.(11), the matrix $\Theta^{(0)}=\left(\theta_{i j}^{(0)}\right)_{n \times n}$ is derived:

$$
\Theta^{(0)}=\left[\begin{array}{llllll}
0 & 0.8807 & 1.0397 & 1.2619 & 1.2182 & 0.9141 \\
0.8807 & 0 & 1.2758 & 1.2952 & 1.2515 & 0.6779 \\
1.0397 & 1.2758 & 0 & 1.1848 & 1.6133 & 0.5493 \\
1.2619 & 1.2952 & 1.1848 & 0 & 1.6145 & 1.0591 \\
1.2182 & 1.2515 & 1.6133 & 1.6145 & 0 & 0.8613 \\
0.9141 & 0.6779 & 0.5493 & 1.0591 & 0.8613 & 0
\end{array}\right]
$$

Step 6. The largest value in $\Psi^{(0)}$ is $\psi_{14}^{(0)}=\psi_{41}^{(0)}=\psi_{16}^{(0)}=\psi_{61}^{(0)}=\psi_{46}^{(0)}=\psi_{64}^{(0)}=1$. Meanwhile, the largest value in the matrix $\Theta^{(0)}$ is $\theta_{45}^{(0)}=\theta_{54}^{(0)}=1.6145$. Because $r_{16}^{(0)}$ and $r_{45}^{(0)}$ are different elements, $r_{16}^{(0)}$ is regarded as the most inconsistent element. 
Step 7. By Eq.(17), $r_{16}^{\prime(0)} / r_{61}^{\prime(0)}=2$ and it is closest to $0.7 / 0.3$ in Table III. Then $r_{16}^{\prime(0)}=0.7$.

Step 8. According to Eq.(18), the improved FPR $R_{4}^{(1)}$ is obtained:

$$
R_{4}^{(1)}=\left[\begin{array}{llllll}
0.5 & 0.3 & 0.3 & 0.7 & 0.8 & 0.7 \\
0.7 & 0.5 & 0.2 & 0.7 & 0.8 & 0.6 \\
0.7 & 0.8 & 0.5 & 0.7 & 0.7 & 0.8 \\
0.3 & 0.3 & 0.3 & 0.5 & 0.9 & 0.7 \\
0.2 & 0.2 & 0.3 & 0.1 & 0.5 & 0.4 \\
0.3 & 0.4 & 0.2 & 0.3 & 0.6 & 0.5
\end{array}\right]
$$

Step 9. By Eq.(8), it is $w^{(1)}=(0.1248,0.1994,0.3408,0.1883,0.0498,0.0969)^{T}$. By Eq.(7), it is $G C I\left(R_{4}^{(1)}\right)=0.4747$ $>\overline{G C I\left(R_{4}\right)}$ and it is still of not acceptable MC.

Step 10. By Algorithm 2, the matrix $\Psi^{(1)}=\left(\psi_{i j}^{(1)}\right)_{6 \times 6}$ is:

$$
\Psi^{(1)}=\left[\begin{array}{cccccc}
0 & 0 & 0 & 0 & 0 & 0 \\
0 & 0 & 0 & 0 & 0 & 0 \\
0 & 0 & 0 & 0 & 0 & 0 \\
0 & 0 & 0 & 0 & 0 & 0 \\
0 & 0 & 0 & 0 & 0 & 0 \\
0 & 0 & 0 & 0 & 0 & 0
\end{array}\right]
$$

Step 11. By Eq.(16), $\psi\left(R_{4}^{(1)}\right)=0$. Thus, $R_{4}^{(1)}$ is OC.

Step 12. By Eqs.(9) and (11), the matrix $\Theta^{(1)}=\left(\theta_{i j}^{(1)}\right)_{n \times n}$ is constructed:

$$
\Theta^{(1)}=\left[\begin{array}{llllll}
0 & 1.0925 & 0.9820 & 1.0500 & 1.0063 & 0.6446 \\
1.0925 & 0 & 1.2758 & 1.2952 & 1.2515 & 0.8898 \\
0.9820 & 1.2758 & 0 & 1.1848 & 1.6133 & 0.4916 \\
1.0500 & 1.2952 & 1.1848 & 0 & 1.6145 & 0.8473 \\
1.0063 & 1.2515 & 1.6133 & 1.6145 & 0 & 0.6495 \\
0.6446 & 0.8898 & 0.4916 & 0.8473 & 0.6495 & 0
\end{array}\right]
$$

Step 13. The largest value in the matrix $\Theta^{(1)}$ is $\theta_{45}^{(1)}=1.6145$. Thus, the corresponding $r_{45}^{(1)}$ in $R_{4}^{(1)}$ is the multiplicative inconsistent element.

Step 14. By Eq.(17), $r_{45}^{\prime(1)} / r_{54}^{\prime(1)}=1.7908$, which is closest to $0.6 / 0.4$ in Table III. Then $r_{45}^{\prime(1)}=0.6$.

Step 15. $r_{45}^{(1)}$ and $r_{45}^{(1)}$ satisfy $\log \left(r_{45}^{(1)} / r_{54}^{(1)}\right) \cdot \log \left(r_{45}^{\prime(1)} / r_{54}^{\prime(1)}\right)>0$, hence the value of $r_{45}^{\prime(1)}$ does not modify the OC.

Step 16. By Eq.(18), the new improved FPR $R_{4}^{(2)}$ is: 


$$
R_{4}^{(2)}=\left[\begin{array}{llllll}
0.5 & 0.3 & 0.3 & 0.7 & 0.8 & 0.7 \\
0.7 & 0.5 & 0.2 & 0.7 & 0.8 & 0.6 \\
0.7 & 0.8 & 0.5 & 0.7 & 0.7 & 0.8 \\
0.3 & 0.3 & 0.3 & 0.5 & 0.6 & 0.7 \\
0.2 & 0.2 & 0.3 & 0.4 & 0.5 & 0.4 \\
0.3 & 0.4 & 0.2 & 0.3 & 0.6 & 0.5
\end{array}\right]
$$

Step 17. By Eq.(8), it is $w^{(2)}=(0.1803,0.2031,0.3471,0.1155,0.0684,0.0856)^{T}$. By Eq.(7), $G C I\left(R_{4}^{(2)}\right)=0.3030$ $<\overline{G C I\left(R_{4}\right)}$, and $R_{4}^{(2)}$ is of acceptable MC. Thus, $R_{4}^{(2)}$ is MC and OC.

The following ranking of alternatives is obtained: $x_{3} \succ x_{2} \succ x_{1} \succ x_{4} \succ x_{6} \succ x_{5}$, which coincides with the improved FPR $R_{4}^{(2)}$.

According to $\mathrm{Xu}$, et al. [29] 's method, the following induced matrix $C^{\prime}$ and improved matrix $R_{4}^{\prime}$ are obtained:

$$
C^{\prime}=\left[\begin{array}{llllll}
1 & 2.1667 & 1.5764 & 0.5539 & 1.4152 & 1.4965 \\
0.5893 & 1 & 3.0595 & 0.8416 & 1.7153 & 1.7346 \\
1.3036 & 0.5521 & 1 & 1.5299 & 4.0714 & 1.0208 \\
2.5910 & 2.5514 & 2.1733 & 1 & 0.4802 & 0.9762 \\
1.7169 & 1.8757 & 0.4826 & 4.0119 & 1 & 1.0142 \\
1.0077 & 0.7944 & 1.1859 & 1.8349 & 1.4193 & 1
\end{array}\right] ; \quad R_{4}^{\prime}=\left[\begin{array}{llllll}
0.5 & 0.3 & 0.3 & 0.7 & 0.8 & 0.6 \\
0.7 & 0.5 & 0.2 & 0.7 & 0.8 & 0.6 \\
0.7 & 0.8 & 0.5 & 0.7 & 0.9 & 0.8 \\
0.3 & 0.3 & 0.3 & 0.5 & 0.9 & 0.7 \\
0.2 & 0.2 & 0.1 & 0.1 & 0.5 & 0.4 \\
0.4 & 0.4 & 0.2 & 0.3 & 0.6 & 0.5
\end{array}\right] \text {. }
$$

The priority vector of $R_{4}^{\prime}$ is $w=(0.1532,0.1857,0.3974,0.1423,0.0370,0.0843)^{T}$ and $G C I\left(R_{4}^{\prime}\right)=0.3097<\overline{G C I\left(R_{4}\right)}$. In each iteration, a pair of elements is adjusted. Observing the induced matrix $C^{\prime}$, since $c_{35} \neq c_{53}, r_{35}$ is regarded as the most multiplicative inconsistent element while $r_{53}$ is not the most multiplicative inconsistent element. Thus, it is unreasonable to adjust $r_{35}$ and $r_{53}$. However, in Algorithm 2, $\Theta$ is a symmetrical matrix, which can easily identify a pair of multiplicative inconsistent elements. Moreover, the proposed method derives a modified FPR with $G C I\left(R_{4}^{(2)}\right)=0.3030$, and therefore with better MC. It means that adjusting $r_{45}$ is more effective than adjusting $r_{35}$.

In comparison with Xia, et al. [13]'s method, for values $\overline{G C I\left(R_{4}\right)}=0.37, \theta=0.1$ ( $\theta$ is a parameter in Xia, et al. [13]'s method), the following matrix is derived:

$$
R_{4}^{(2)}=\left[\begin{array}{llllll}
0.5000 & 0.3240 & 0.3021 & 0.6654 & 0.7920 & 0.5220 \\
0.6760 & 0.5000 & 0.2272 & 0.6764 & 0.8000 & 0.6144 \\
0.6979 & 0.7728 & 0.5000 & 0.6981 & 0.7411 & 0.7960 \\
0.3346 & 0.3236 & 0.3019 & 0.5000 & 0.8800 & 0.6841 \\
0.2080 & 0.2000 & 0.2589 & 0.1200 & 0.5000 & 0.3882 \\
0.4780 & 0.3856 & 0.2040 & 0.3159 & 0.6118 & 0.5000
\end{array}\right]
$$

This matrix has a larger value $G C I\left(R_{4}^{(2)}\right)=0.3312<\overline{G C I\left(R_{4}\right)}$ than the corresponding one for the matrix derived from the proposed method. In addition, the proposed method retains most of the original judgments. It only needs to adjust 4 
elements. Therefore, the proposed method is not only simpler and effective but also has better consistency and retains more original preference information.

\section{B. Monte Carlo Simulation and Further Discussion}

This subsection further discusses the effectiveness of the proposed methods. Monte Carlo simulations depends on the random sampling to generate the results, and is an important method to model uncertainty phenomena. In order to do this, 1,000 FPRs for different dimension ranging from 3 to 9 are randomly generated and their consistencies (ordinal and/or multiplicative) are improved using the proposed method. When randomly generating FPRs, the continuous domain $(0,1)$ is used and therefore the values of entries will not be in the discrete scale $S_{[0.1,0.9]}$. Thus, in the adjustment process, the adjusted values are in the domain $(0,1)$. The average numbers of iterations of Algorithm 3 with different thresholds for different dimension values $n$ (of Table I) are provided in Table IV and plotted in Fig. 13. For $n=3$, the average number of iterations is lower than 1, denoting that some of the randomly generated FPRs are of OC and MC (these FPRs are not needed to be revised); otherwise, only one iteration is required to achieve the OC and MC. From Table IV and Fig. 3, it is clear that the average number of iterations increases as $n$ increases. The percentages of the entries changed in the matrices are listed in Table $\mathrm{V}$ against their dimension value $n$, with an increasing tendency observed. In any case, the majority of the original values are not modified (largest percentage change value is $36.56 \%$ ) with the proposed method. This also indicates the effectiveness of the proposed method.

TABLE IV. THE AVERAGE ITERATIONS FOR DIFFERENT $n$

\begin{tabular}{c|c|c|c|c|c|c|c}
\hline$n$ & 3 & 4 & 5 & 6 & 7 & 8 & 9 \\
\hline iterations & 0.758 & 1.879 & 3.332 & 5.417 & 7.88 & 11.057 & 14.807 \\
\hline
\end{tabular}

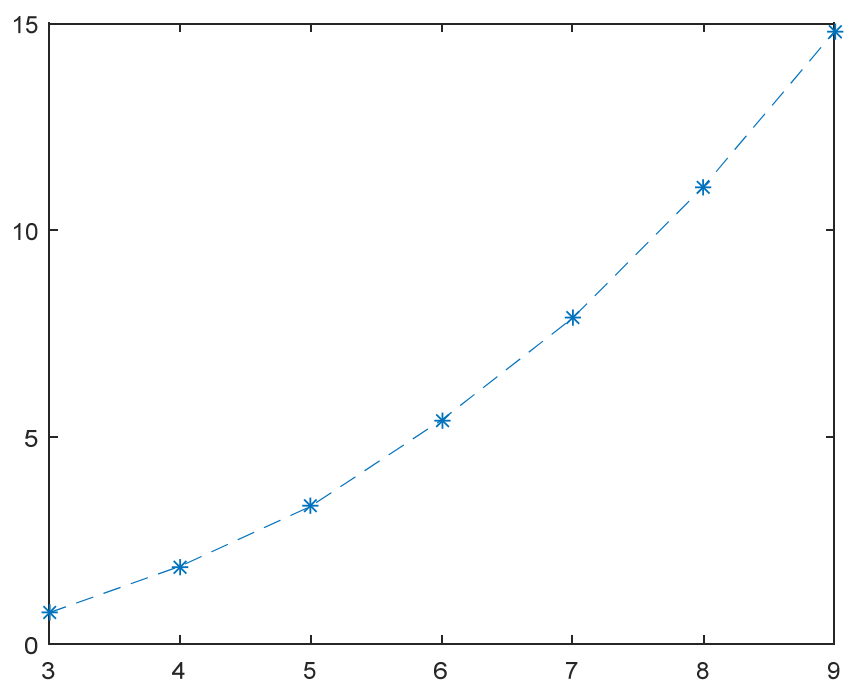

Fig. 3. The average number of iterations of the proposed method

TABLE V. THE PERCENTAGE OF THE VALUES CHANGED FOR DIFFERENT $n$

\begin{tabular}{c|c|c|c|c|c|c|c}
\hline$n$ & 3 & 4 & 5 & 6 & 7 & 8 & 9 \\
\hline percentage & $16.84 \%$ & $23.49 \%$ & $26.66 \%$ & $30.09 \%$ & $32.16 \%$ & $34.55 \%$ & $36.56 \%$ \\
\hline
\end{tabular}




\section{CONCLUSIONS}

In this paper, algorithms to rectify the multiplicative and ordinal inconsistencies for FPRs have been proposed. Firstly, we have analyzed the MC property of FPRs. Geometric consistency has been applied to measure the MC degree of an FPR. The approximated thresholds of GCI for MPRs were extended to the case of FPRs to test whether an FPR is of acceptable MC. To improve the level of MC:

- First, the degree of inconsistency is measured with $G C I$, and then a matrix $\Theta=\left(\theta_{i j}\right)_{n \times n}$ is established to identify the multiplicative inconsistent elements.

- Secondly, we presented the ordinal inconsistent conditions of FPRs. An effective algorithm to find the ordinally inconsistent elements was developed. Meanwhile, we proposed an approach based on the matrix $\psi(R)$ to measure the level of OC for FPRs.

- Thirdly, we designed an integrated method to improve simultaneously the OC and MC of an FPR.

- Finally, we demonstrated the validity of our proposed integrated consistency improvement method with its superior performance against existing methods using both examples that represent the four different scenarios of ordinal and multiplicative inconsistencies and simulation experiments. The following advantages noticed:

(1) Only the MC is considered in [13], and only the OC is considered in [15]. Our proposed method can improve multiplicative and OC simultaneously.

(2) Our method is simple and straightforward. In the process of improving of MC and OC, most of the original judgements are kept unchanged. This is possible because, at each iteration of the proposed method, inconsistent elements are accurately located and only one pair of preference values are modified. In contrast, other existing methods [13, 30, 32] change all the original judgments given by DM.

(3) The developed method can be applied not only to strict FPRs but also to non-strict FPRs. However, Ma, et al. [30] can only be applied to strict FPRs.

In the future, we will study the MC and OC for incomplete FPRs [25, 36-41] and will consider the application of the proposed method in group consensus models [42-50].

\section{REFERENCES}

[1] N. Capuano, F. Chiclana, H. Fujita, E. Herrera-Viedma, and V. Loia, "Fuzzy group decision making with incomplete information guided by social influence," IEEE Transactions on Fuzzy Systems, vol. 26, no. 3, pp. 1704-1718, 2018.

[2] W. Liu, Y. C. Dong, F. Chiclana, F. J. Cabrerizo, and E. Herrera-Viedma, "Group decision-making based on heterogeneous preference relations with self-confidence," Fuzzy Optimization and Decision Making, vol. 16, no. 4, pp. 429-447, 2017.

[3] H. L. Yin, R. Li, and J. Lan, "Pairwise comparison based ranking vector approach to estimation performance ranking," IEEE Transactions on Systems, Man, and Cybernetics: Systems, vol. 48, no. 6, pp. 942-953, 2018.

[4] T. L. Saaty, "The Analytic Hierarchy Process," McGraw-Hill, New York, 1980.

[5] S. Siraj and L. Mikhailovb, "A heuristic method to rectify intransitive judgments in pairwise comparison matrices," European Journal of Operational Research, vol. 216, no. 2, pp. 420-428, 2012. 
[6] Z. S. Xu and C. P. Wei, "A consistency improving method in the analytic hierarchy process," European Journal of Operational Research, vol. 116, no. 2, pp. 443-449, 1999.

[7] F. Herrera, E. Herrera-Viedma, and F. Chiclana, "Multiperson decision-making based on multiplicative preference relations," European Journal of Operational Research, vol. 129, no. 2, pp. 372-385, 2001.

[8] D. Ergu, G. Kou, and P. Yi, "A simple method to improve the consistency ratio of the pair-wise comparison matrix in ANP," European Journal of Operational Research, vol. 213, no. 1, pp. 246-259, 2011.

[9] S. Siraj, L. Mikhailov, and J. A. Keane, "Contribution of individual judgments toward inconsistency in pairwise comparisons," European Journal of Operational Research, vol. 242, pp. 557-567, 2015.

[10] E. Herrera-Viedma, F. Herrera, F. Chiclana, and M. Luque, "Some issues on consistency of fuzzy preference relations," European Journal of Operational Research, vol. 154, no. 1, pp. 98-109, 2004.

[11] T. Tanino, "Fuzzy preference orderings in group decision making," Fuzzy Sets \& Systems, vol. 12, no. 2, pp. 117-131, 1984.

[12] F. Chiclana, F. Herrera, and E. Herrera-Viedma, "Integrating three representation models in fuzzy multipurpose decision making based on fuzzy preference relations," Fuzzy Sets and Systems, vol. 97, no. 1, pp. 33-48, 1998.

[13] M. M. Xia, Z. S. Xu, and J. Chen, "Algorithms for improving consistency or consensus of reciprocal [0,1]-valued preference relations," Fuzzy Sets and Systems, vol. 216, pp. 108-133, 2013.

[14] F. J. Cabrerizo, J. A. Morente-Molinera, W. Pedrycz, A. Taghavi, and E. Herrera-Viedma, "Granulating linguistic information in decision making under consensus and consistency," Expert Systems with Applications, vol. 99, pp. 83-92, 2018.

[15] Y. J. Xu, R. Patnayakuni, and H. M. Wang, "The ordinal consistency of a fuzzy preference relation," Information Sciences, vol. 224, pp. 152-164, 2013.

[16] Y. J. Xu, F. Herrera, and H. M. Wang, "A distance-based framework to deal with ordinal and additive inconsistencies for fuzzy reciprocal preference relations," Information Sciences, vol. 328, pp. 189-205, 2016.

[17] M. Kwiesielewicz and E. van Uden, "Inconsistent and contradictory judgements in pairwise comparison method in the AHP," Computers \& Operations Research, vol. 31, no. 5, pp. 713-719, 2004.

[18] H. L. Li and L. C. Ma, "Detecting and adjusting ordinal and cardinal inconsistencies through a graphical and optimal approach in AHP models," Computers \& Operations Research, vol. 34, no. 3, pp. 780-798, 2007.

[19] Y. J. Xu, F. Ma, and F. Herrera, "Revisiting inconsistent judgments for incomplete fuzzy linguistic preference relations: Algorithms to identify and rectify ordinal inconsistencies," Knowledge-Based Systems, vol. 163, pp. 305-319, 2019.

[20] F. Chiclana, E. Herrera-Viedma, S. Alonso, and F. Herrera, "Cardinal consistency of reciprocal preference relations: a characterization of multiplicative transitivity," IEEE Transactions on Fuzzy Systems, vol. 17, no. 1, pp. 14-23, 2009.

[21] T. Tanino, "Fuzzy preference relations in group decision making," in Non-Conventional Preference Relations in Decision Making. Lecture Notes in Economics and Mathematical Systems, vol. 301, J. Kacprzyk and M. Roubens, Eds.: Springer Berlin Heidelberg, 1988 , pp. 54-71.

[22] C. C. Li, Y. C. Dong, Y. J. Xu, F. Chiclana, E. Herrera-Viedma, and F. Herrera, "An overview on managing additive consistency of reciprocal preference relations for consistency-driven decision making and fusion: Taxonomy and future directions," Information Fusion, vol. 52, pp. 143-156, 2019.

[23] B. D. Baets and H. D. Meyer, "Transitivity frameworks for reciprocal relations: cycle-transitivity versus FG -transitivity," Fuzzy Sets and Systems, vol. 152, no. 2, pp. 249-270, 2005.

[24] R. D. Luce and P. Suppes, "Preferences, utility and subject probability," in Handbook of Mathematical Psychology, vol. III, R. D. Luce, R. Bush, and E. Galanter, Eds. New York: Wiley, 1965, pp. 249-410.

[25] Y. J. Xu, J. N. D. Gupta, and H. M. Wang, "The ordinal consistency of an incomplete reciprocal preference relation," Fuzzy Sets and Systems, vol. 246, no. 4 , pp. $62-77,2014$.

[26] Y. J. Xu, Q. L. Da, and L. H. Liu, "Normalizing rank aggregation method for priority of a fuzzy preference relation and its effectiveness," International Journal of Approximate Reasoning, vol. 50, no. 8, pp. 1287-1297, 2009.

[27] Z. B. Wu and J. P. Xu, "A concise consensus support model for group decision making with reciprocal preference relations based on deviation measures," Fuzzy Sets and Systems, vol. 206, no. 11, pp. 58-73, 2012.

[28] F. Y. Meng, Q. X. An, C. Q. Tan, and X. H. Chen, "An approach for group decision making with interval fuzzy preference relations based on additive consistency and consensus analysis," IEEE Transactions on Systems, Man, and Cybernetics: Systems, vol. 47, no. 8, pp. 2069-2082, 2017. 
[29] Y. J. Xu, Q. Q. Wang, F. J. Cabrerizo, and E. Herrera-Viedma, "Methods to improve the ordinal and multiplicative consistency for reciprocal preference relations," Applied Soft Computing, vol. 67, pp. 479-493, 2018.

[30] J. Ma, Z.-P. Fan, Y.-P. Jiang, J.-Y. Mao, and L. Ma, "A method for repairing the inconsistency of fuzzy preference relations," Fuzzy Sets and Systems, vol. 157, no. 1, pp. 20-33, 2006.

[31] J. Aguarón and J. M. A. Moreno-Jiménez, "The geometric consistency index: Approximated thresholds," European Journal of Operational Research, vol. 147, no. 1, pp. 137-145, 2007.

[32] Z. S. Xu and Q. L. Da, "An approach to improving consistency of fuzzy preference matrix," Fuzzy Optimization \& Decision Making, vol. 2, no. 1, pp. 3-12, 2003.

[33] Y. M. Wang and Z. P. Fan, "Group decision analysis based on fuzzy preference relations: Logarithmic and geometric least squares methods," Applied Mathematics and Computation, vol. 194, no. 1, pp. 108-119, 2007.

[34] F. Chiclana, F. Mata, L. Martinez, E. Herrera-Viedma, and S. Alonso, "Integration of a consistency control module within a consensus model," International Journal of Uncertainty, Fuzziness and Knowledge-Based Systems, vol. 16, no. 1, pp. 35-53, 2008.

[35] Z. S. Xu and X. Q. Cai, "Group consensus algorithms based on preference relations," Information Sciences, vol. 181, no. 1, pp. 150-162, 2011.

[36] Y. J. Xu and H. M. Wang, "Eigenvector method, consistency test and inconsistency repairing for an incomplete fuzzy preference relation," Applied Mathematical Modelling, vol. 37, no. 7, pp. 5171-5183, 2013.

[37] E. Herrera-Viedma, S. Alonso, F. Chiclana, and F. Herrera, "A consensus model for group decision making with incomplete fuzzy preference relations," IEEE Transactions on Fuzzy Systems, vol. 15, no. 5, pp. 863-877, 2007.

[38] S. Alonso, F. J. Cabrerizo, F. Chiclana, F. Herrera, and E. Herrera-Viedma, "Group decision making with incomplete fuzzy linguistic preference relations," International Journal of Intelligent Systems, vol. 24, no. 2, pp. 201-222, 2009.

[39] A. Khalid and M. M. Awais, "Incomplete Preference Relations: An upper bound condition," Journal of Intelligent \& Fuzzy Systems, vol. 26, no. 3, pp. 1433-1438, 2014.

[40] A. Khalid and I. Beg, "Incomplete interval valued fuzzy preference relations," Information Sciences, vol. 348, pp. 15-24, 2016.

[41] S. Yao and A. Khalid, "Completing 2-tuple linguistic preference relations based on upper bound condition," Soft Computing, no. 1, pp. 1-13, 2017.

[42] E. Herrera-Viedma, F. Herrera, and F. Chiclana, "A consensus model for multiperson decision making with different preference structures," IEEE Transactions on Systems Man and Cybernetics Part A Systems \& Humans, vol. 32, no. 3, pp. 394-402, 2002.

[43] F. J. Cabrerizo, F. Chiclana, R. Al-Hmouz, A. Morfeq, A. S. Balamash, and E. Herrera-Viedma, "Fuzzy decision making and consensus: challenges," Journal of Intelligent \& Fuzzy Systems, vol. 29, no. 3, pp. 1109-1118, 2015.

[44] Y. C. Dong, G. Q. Zhang, W. C. Hong, and Y. F. Xu, "Consensus models for AHP group decision making under row geometric mean prioritization method," Decision Support Systems, vol. 49, no. 3, pp. 281-289, 2010.

[45] F. J. Cabrerizo, R. Al-Hmouz, A. Morfeq, A. S. Balamash, M. A. Martínez, and E. Herrera-Viedma, "Soft consensus measures in group decision making using unbalanced fuzzy linguistic information," Soft Computing, vol. 21, pp. 3037-3050, 2017.

[46] Y. J. Xu, X. W. Wen, and W. C. Zhang, "A two-stage consensus method for large-scale multi-attribute group decision making with an application to earthquake shelter selection," Computers \& Industrial Engineering, vol. 116, pp. 113-129, 2018.

[47] H. J. Zhang, Y. C. Dong, and X. Chen, "The 2-rank consensus reaching model in the multigranular linguistic multiple-attribute group decision-making," IEEE Transactions on Systems, Man, and Cybernetics-Systems, p. doi: 10.1109/TSMC.2017.2694429, 2018.

[48] G. X. Li, G. Kou, and Y. Peng, "A group decision making model for integrating heterogeneous information," IEEE Transactions on Systems, Man, and Cybernetics: Systems, vol. 48, no. 6, pp. 982-992, 2018.

[49] I. J. Pérez, F. J. Cabrerizo, S. Alonso, and E. Herrera-Viedma, "A new consensus model for group decision making problems with non-homogeneous experts," IEEE Transactions on Systems, Man, and Cybernetics: Systems, vol. 44, no. 4, pp. 494-498, 2014.

[50] Y. T. Liu, Y. C. Dong, H. M. Liang, F. Chiclana, and E. Herrera-Viedma, "Multiple attribute strategic weight manipulation with minimum cost in a group decision making context with interval attribute weights information," IEEE Transactions on Systems, Man and Cybernetics: Systems, p. doi: 10.1109/TSMC.2018.2874942, 2018. 